\title{
From positive geometries to a coaction on hypergeometric functions
}

\author{
Samuel Abreu, ${ }^{a}$ Ruth Britto, ${ }^{b, c, d}$ Claude Duhr, ${ }^{e}$ Einan Gardi ${ }^{f}$ and James Matthew ${ }^{f}$ \\ ${ }^{a}$ Center for Cosmology, Particle Physics and Phenomenology (CP3), \\ Université Catholique de Louvain, \\ 1348 Louvain-La-Neuve, Belgium \\ ${ }^{b}$ School of Mathematics, Trinity College, \\ Dublin 2, Ireland \\ ${ }^{c}$ Hamilton Mathematics Institute, Trinity College, \\ Dublin 2, Ireland \\ ${ }^{d}$ Institut de Physique Théorique, Université Paris Saclay, CEA, CNRS, \\ F-91191 Gif-sur-Yvette cedex, France \\ e Theoretical Physics Department, CERN, \\ Geneva, Switzerland \\ ${ }^{f}$ Higgs Centre for Theoretical Physics, School of Physics and Astronomy, \\ The University of Edinburgh, \\ Edinburgh EH9 3FD, Scotland, U.K. \\ E-mail: samuel.abreu@uclouvain.be, britto@maths.tcd.ie, \\ claude.duhr@cern.ch, einan.gardi@ed.ac.uk, james.matthew@ed.ac.uk
}

ABSTRACT: It is well known that Feynman integrals in dimensional regularization often evaluate to functions of hypergeometric type. Inspired by a recent proposal for a coaction on one-loop Feynman integrals in dimensional regularization, we use intersection numbers and twisted homology theory to define a coaction on certain hypergeometric functions. The functions we consider admit an integral representation where both the integrand and the contour of integration are associated with positive geometries. As in dimensionallyregularized Feynman integrals, endpoint singularities are regularized by means of exponents controlled by a small parameter $\epsilon$. We show that the coaction defined on this class of integral is consistent, upon expansion in $\epsilon$, with the well-known coaction on multiple polylogarithms. We illustrate the validity of our construction by explicitly determining the coaction on various types of hypergeometric ${ }_{p+1} F_{p}$ and Appell functions.

KEYwords: Scattering Amplitudes, Perturbative QCD

ARXIV EPRINT: 1910.08358 


\section{Contents}

1 Introduction 1

2 The coaction on MPLs and resummation of the $\epsilon$ expansion 4

2.1 The coaction on MPLs 5

2.2 Resummation of the $\epsilon$ expansion 6

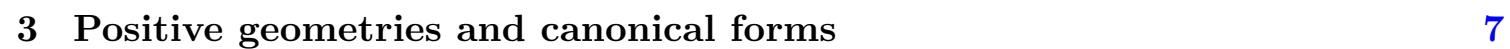

3.1 A class of integrals 8

$\begin{array}{ll}3.2 & \text { Integrals and positive geometries }\end{array}$

$\begin{array}{lll}3.3 & \text { Pairings of cycles and forms } & 12\end{array}$

4 A coaction on integrals $\quad 14$

5 Gauss' hypergeometric function ${ }_{2} F_{1} \quad 16$

$\begin{array}{ll}5.1 \text { The coaction in the basis of canonical forms } & 17\end{array}$

$\begin{array}{ll}5.2 \text { Coaction in an orthonormal basis } & 18\end{array}$

$\begin{array}{ll}5.3 \text { Coaction of a degenerate }{ }_{2} F_{1} & 19\end{array}$

6 One-dimensional integrals: Lauricella functions $F_{D}^{(n)} \quad 21$

6.1 The Appell $F_{1}$ function 22

$\begin{array}{ll}6.2 \text { Coaction for generic Lauricella } F_{D}^{(n)} & 23\end{array}$

7 Two-dimensional integrals: Appell functions $\quad 24$

$\begin{array}{lll}7.1 & \text { The Appell } F_{1} \text { function as a double integral } & 25\end{array}$

$\begin{array}{lll}7.2 & \text { The Appell } F_{3} \text { function } & 28\end{array}$

7.3 The Appell $F_{2}$ function $\quad 30$

7.4 The Appell $F_{4}$ function 33

8 The generalized hypergeometric function ${ }_{p+1} F_{p} \quad 36$

9 Summary and discussion $\quad 38$

\section{Introduction}

Feynman integrals are a cornerstone of perturbative quantum field theory and they are ubiquitous when evaluating higher orders in the perturbative series. As such, having efficient tools for their evaluation and a thorough understanding of their mathematical properties is of great importance. For this reason, Feynman integrals and their mathematical structure are an active field of study both in mathematics and physics. 
It follows from unitarity that Feynman integrals must be transcendental functions, as they must have nonvanishing discontinuities stemming from logarithmic branch cuts. In integer dimensions, the class of transcendental functions that can arise is further constrained to be periods $[1,2]$, which are integrals of algebraic functions over domains defined by inequalities between algebraic functions [3]. Periods are interesting objects in their own right in mathematics, and it is known that they can be equipped with a lot of algebraic structure. Of particular interest for this paper will be the so-called coaction, see for example ref. [4]. It was shown in ref. [5] that, quite generically, this algebraic structure and the coaction are inherited by the Feynman integrals themselves. Understanding these structures in detail may open the way to a novel understanding of perturbative quantum field theory. For first applications in a physics context, see, e.g., refs. [6-8].

While the algebraic structures on Feynman integrals introduced in ref. [5] apply in a broad range of cases, they ignore a key aspect of Feynman integrals arising in physicallyrelevant perturbative quantum field theories, namely the unavoidable fact that these involve divergent integrals in four space-time dimensions. In order to make sense of divergent integrals one needs to introduce a suitable regulator. A consistent framework for regularizing the divergences is provided by dimensional regularization, where the integral is computed in $D=4-2 \epsilon$ dimensions. The Feynman integral is then a meromorphic function of $\epsilon$, as can be seen, for instance, from the so-called Feynman-parametric representation. Singularities may occur at rational values of $\epsilon$, and those at $\epsilon=0$ encode the divergences of the integrals in the four-dimensional limit. The route leading from divergent Feynman integrals to finite physical quantities is rather involved: ultraviolet singularities are eliminated in the process of renormalization, while infrared ones cancel in infrared-finite observables. As a consequence, dimensionally-regularized Feynman integrals are an integral part of most modern approaches to compute higher-order corrections in perturbation theory.

As functions of $\epsilon$, Feynman integrals are not periods in the same sense as considered above. ${ }^{1}$ Instead, it is the coefficients in their Laurent expansion in $\epsilon$ that are periods [2]. Algebraic structures, such as the coaction, that have been defined for periods do not directly extend to dimensionally-regularized integrals, and one can only apply the coaction order by order in $\epsilon$. However, if one believes that the coaction is an intrinsic property of the Feynman integrals themselves, one might expect that there should be a way to extend it beyond the formalism developed for periods. First steps in that direction were taken in [9, 10], where we conjectured a formula for a coaction that maps integrals into pairs of integrals obtained from a basis of integrands $\left\{\omega_{i}\right\}$ and integration contours $\left\{\gamma_{j}\right\}$ according to

$$
\Delta\left(\int_{\gamma} \omega\right)=\sum_{i j} c_{i j} \int_{\gamma} \omega_{i} \otimes \int_{\gamma_{j}} \omega .
$$

More precisely, the $\left\{\omega_{i}\right\}$ are forms that generate the cohomology group associated with the integral on the left-hand side, and the $\left\{\gamma_{j}\right\}$ are cycles that generate the corresponding homology group. In refs. $[9,10]$ the matrix $c_{i j}$ was computed using an operation called

\footnotetext{
${ }^{1}$ However, dimensionally regularized Feynman integrals define twisted periods. Indeed, twisted (co)homology theory will play an important role in our framework.
} 
semi-simple projection. In the case of one-loop integrals, the first entry of the tensor in the coaction was identified as the integral associated with a contraction of the original Feynman graph, and the second entry as a cut of the Feynman integral [11, 12], leading to an elegant diagrammatic representation for the coaction. One-loop Feynman integrals have the special property that the periods that appear in their Laurent expansion in $\epsilon$ are all multiple polylogarithms (MPLs), and, remarkably, the coaction obtained from eq. (1.1) was shown to be consistent with the expansion in $\epsilon$. More precisely, it was observed that if both sides of the equality in eq. (1.1) are expanded in $\epsilon$, then the coaction in eq. (1.1) reproduces the coaction on MPLs.

It is well known that hypergeometric-type integrals (see, e.g., ref. [13]) appear when evaluating Feynman integrals in dimensional regularization. These functions depend on a set of parameters and a set of variables. For instance, the well-known Gauss hypergeometric function ${ }_{2} F_{1}(\alpha, \beta ; \gamma ; x)$ depends on the parameters $\alpha, \beta, \gamma$ and the variable $x$; in the Euler-type integral representation we will be using, given in eq. (2.12) below, the former parametrize the exponents governing the powers of polynomial functions of the latter. In the context of Feynman integrals, the parameters are linear in the dimensional regulator $\epsilon$, and the variables depend on the kinematics of the Feynman diagram. The specific type of hypergeometric function also depends on the diagram considered. Let us recall a few examples from the literature: for one-loop integrals in general kinematics one finds the ${ }_{2} F_{1}$ function in two-point functions (see e.g. the appendix of ref. [10]), the so-called Appell $F_{1}$ function in three-point functions and the Lauricella-Saran $F_{S}$ or $F_{N}$ functions [14] in four-point functions (see e.g. refs. $[15,16]$ ). Higher-point integrals have not been computed explicitly in general kinematics as a function of $\epsilon$, but for example the massless pentagon evaluates to Appell $F_{3}$ functions [17]. Beyond one loop, one also finds that Feynman integrals evaluate to similar classes of hypergeometric functions. For instance, two-loop sunrise integrals evaluate to either ${ }_{2} F_{1}$, Appell $F_{2}$ or Appell $F_{4}$ functions, depending on the configuration of the masses of the propagators, see e.g. ref. [18]. For other examples of hypergeometric functions appearing in the calculation of Feynman integrals, including very recent work, see refs. [19-25]. These examples illustrate the fact that hypergeometrictype integrals are the functions we must understand when studying Feynman integrals in dimensional regularization.

Building on the results of refs. [9, 10], where one-loop Feynman integrals were observed to admit a diagrammatic coaction, valid to all orders in the dimensional regulator, it is natural to expect that one could define a coaction that acts on the relevant functions, independently of whether they appear in a Feynman integral. By imposing restrictions on the form of the parameters, we will focus on cases where the hypergeometric functions expand to MPLs, so that we can explicitly verify that the coaction we obtain from (1.1) reduces to the coaction on MPLs upon expansion in $\epsilon$. Constructing such a coaction is the main goal of this paper. It is important both for the study of hypergeometric-type integrals and in view of the possible extension of the diagrammatic coaction beyond one loop.

The starting point for constructing the coaction in all cases of hypergeometric functions we will address is their integral representation. We find the concept of positive geometries [26] very useful to study these integrals, because it allows us to find convenient 
bases of for the homology and cohomology groups of the corresponding integral representations. With these bases, we can directly use the general formula in eq. (1.1) to obtain a coaction on the corresponding integrals.

In this paper, we also introduce a new feature in the construction of the coaction in eq. (1.1). The matrix $c_{i j}$ is constructed by computing the matrix of intersection numbers between the generators of the cohomology group $\omega_{i}$ and a set of forms $\Omega\left(\gamma_{i}\right)$ which, under certain conditions, can be constructed in a canonical way from the contours $\gamma_{i}$. This is always possible to do for integrals defined by positive geometries, which includes all examples we will address in this paper and for which there is an explicit way of computing the forms $\Omega\left(\gamma_{i}\right)$. Because we consider functions prior to expansion in $\epsilon$, the integrands are themselves multi-valued functions. This implies that we cannot use standard (co)homology theory to construct our bases of forms and cycles. Instead, we must use the framework of 'twisted (co)homology' [27] which is well known in the mathematics literature. Recently, these tools have been applied in several areas of theoretical physics such as in string theory [28, 29] or in the study of the integration-by-parts relations satisfied by Feynman integrals [30-32]. Compared with the construction of refs. $[9,10]$, where the normalization was based on a semi-simple projection, the approach we present here has the advantage of treating the generators of the homology and cohomology groups on the same footing, in the sense that the matrix $c_{i j}$ can be viewed as a change of basis of the generators of either group.

We would like to mention that, following discussions about the content of refs. $[9,10,33-35]$ and parts of the content of the present paper, the authors of ref. [36] have initiated a rigorous mathematical treatment of the concepts presented in this paper, specialized to a class of one-dimensional integrals representing Lauricella $F_{D}$ functions.

The paper is organized as follows. In section 2 we summarize the coaction on MPLs and give first examples of a coaction acting on an unexpanded function of $\epsilon$, obtained by resumming the Laurent series of the integrals. In section 3 we discuss positive geometries in order to define the type of integrals that we will consider in this paper, and we very briefly introduce the elements of twisted (co)homology theory that will be relevant for this paper. Section 4 contains the main result of the paper, namely the formula for a coaction that acts on unexpanded $\epsilon$-dependent integrals, while being consistent with the Laurent expansion in $\epsilon$. The remaining sections contain examples in a variety of hypergeometric-type integrals. We first discuss in detail Gauss' hypergeometric function ${ }_{2} F_{1}$ in section 5 . In section 6 we discuss a larger class of one-dimensional integrals depending on several variables, namely the Lauricella $F_{D}$ functions. In section 7 we discuss two-dimensional integrals, focusing on the Appell functions $F_{1}, F_{2}, F_{3}$ and $F_{4}$. Finally, in section 8 we discuss generic ${ }_{p+1} F_{p}$ hypergeometric functions, which are given by a $p$-dimensional integral. In section 9 we summarize and discuss our results.

\section{The coaction on MPLs and resummation of the $\epsilon$ expansion}

In this section we give a brief review of MPLs and their coaction to establish our notation. We then discuss some simple examples of Feynman integrals in dimensional regularization and hypergeometric functions where one can 'resum' the Laurent series around $\epsilon=0$ to obtain a coaction that is consistent with the Laurent expansion. 


\subsection{The coaction on MPLs}

When considered order-by-order in dimensional regularization, many multi-loop Feynman integrals can be evaluated in terms of MPLs, defined by the iterated integrals [37]

$$
G\left(a_{1}, \ldots, a_{n} ; z\right)=\int_{0}^{z} \frac{d t}{t-a_{1}} G\left(a_{2}, \ldots, a_{n} ; t\right)
$$

where the $a_{i}$ and $z$ are (algebraic) complex numbers. In the case where all $a_{i}=0$, the integral in eq. (2.1) diverges, and instead we define

$$
G\left(\overrightarrow{0}_{n} ; z\right)=\frac{1}{n !} \log ^{n} z, \quad \overrightarrow{0}_{n}=(\underbrace{0, \ldots, 0}_{n \text { times }}) .
$$

MPLs are well studied in both the mathematics and physics literature (see, e.g., ref. [38] and references therein). In particular, they can be endowed with a coaction [37, 39, 40], which we denote here by $\Delta$. Roughly speaking, the coaction associates to an MPL a linear combination of tensor products of these functions. For example, the coactions of the logarithm in eq. (2.2) or of the classical polylogarithm $\operatorname{Li}_{n}(z)=-G\left(\overrightarrow{0}_{n-1}, 1 ; z\right)$ are given by

$$
\begin{aligned}
& \Delta\left(\log ^{n} z\right)=\sum_{k=0}^{n}\left(\begin{array}{l}
n \\
k
\end{array}\right) \log ^{n-k} z \otimes \log ^{k} z, \\
& \Delta\left(\operatorname{Li}_{n}(z)\right)=1 \otimes \operatorname{Li}_{n}(z)+\sum_{k=0}^{n-1} \operatorname{Li}_{n-k}(z) \otimes \frac{\log ^{k} z}{k !} .
\end{aligned}
$$

The formula for the coaction of a general MPL is more involved, and we refer to the literature for a discussion of the general case [37, 39, 40].

An important feature of the coaction on MPLs is that the second factor of each tensor is interpreted modulo its branch cuts. Since all discontinuities of MPLs are proportional to powers of $i \pi$, this is equivalent to setting to zero all factors of $i \pi$ in the second factor of each tensor in the coaction. The coaction also operates nontrivially on transcendental constants obtained by specialising the arguments of the MPLs to some special values. In particular, at $z=1$ the classical polylogarithms reduce to zeta values, $\zeta_{n}=\operatorname{Li}_{n}(1)$. For $n$ odd, the coaction of $\zeta_{n}$ is simply obtained by specialising eq. (2.3) to $z=1$,

$$
\Delta\left(\zeta_{n}\right)=\zeta_{n} \otimes 1+1 \otimes \zeta_{n}, \quad n \text { odd }
$$

For $n$ even, the situation is more subtle, and we have to define $[40,41]$

$$
\Delta\left(\zeta_{n}\right)=\zeta_{n} \otimes 1, \quad n \text { even }
$$

and more generally

$$
\Delta(i \pi)=i \pi \otimes 1
$$

These definitions are consistent with the fact that we have to work modulo factors of $i \pi$ in the second factor. 


\subsection{Resummation of the $\epsilon$ expansion}

When working in dimensional regularization, MPLs appear as the Laurent coefficients in the $\epsilon$ expansion, and we can only consider the coaction order by order in the expansion. A natural question to ask is if one can 'resum' the Laurent series after acting with $\Delta$ on its coefficients. To illustrate this point, let us consider the simplest Feynman integral, namely the tadpole integral with one massive propagator in $D=2-2 \epsilon$ dimensions,

$$
\begin{aligned}
T\left(m^{2}, \epsilon\right) & =\frac{e^{\gamma_{E} \epsilon}}{i \pi^{D / 2}} \int \frac{d^{D} k}{k-m^{2}}=\frac{e^{\gamma_{E} \epsilon} \Gamma(1+\epsilon)}{\epsilon(1-\epsilon)} m^{-2 \epsilon} \\
& =\frac{1}{\epsilon}+1-\log m^{2}+\epsilon\left(\frac{1}{2} \log ^{2} m^{2}-\log m^{2}+1+\frac{\pi^{2}}{12}\right)+\mathcal{O}\left(\epsilon^{2}\right),
\end{aligned}
$$

where $\gamma_{E}=-\Gamma^{\prime}(1)$ denotes the Euler-Mascheroni constant. We can act with $\Delta$ order by order in the expansion, and it is straightforward to check that at each order the resulting formula is consistent with a 'resummed' coaction,

$$
\Delta\left(T\left(m^{2}, \epsilon\right)\right)=\frac{1}{\epsilon(1-\epsilon)}\left[e^{\gamma_{E} \epsilon} \Gamma(1+\epsilon) m^{-2 \epsilon}\right] \otimes\left[e^{\gamma_{E} \epsilon} \Gamma(1+\epsilon) m^{-2 \epsilon}\right] .
$$

In fact, it is easy to prove the previous formula by using the fact that $\Delta(a \cdot b)=\Delta(a) \cdot \Delta(b)$ as well as

$$
\begin{aligned}
\Delta\left(\frac{1}{\epsilon(1-\epsilon)}\right) & =\frac{1}{\epsilon(1-\epsilon)} 1 \otimes 1, \\
\Delta\left(m^{-2 \epsilon}\right) & =m^{-2 \epsilon} \otimes m^{-2 \epsilon}, \\
\Delta\left[e^{\gamma_{E} \epsilon} \Gamma(1+\epsilon)\right] & =\left[e^{\gamma_{E} \epsilon} \Gamma(1+\epsilon)\right] \otimes\left[e^{\gamma_{E} \epsilon} \Gamma(1+\epsilon)\right] .
\end{aligned}
$$

These formulas are obtained by expanding the argument of $\Delta$ in $\epsilon$ and using the linearity of the coaction. For instance,

$$
\begin{aligned}
\Delta\left(\frac{1}{\epsilon(1-\epsilon)}\right) & =\sum_{k=0}^{\infty} \epsilon^{k-1} \Delta(1)=\sum_{k=0}^{\infty} \epsilon^{k-1}(1 \otimes 1)=\frac{1}{\epsilon(1-\epsilon)} 1 \otimes 1, \\
\Delta\left(m^{-2 \epsilon}\right) & =\sum_{k=0}^{\infty} \frac{(-\epsilon)^{k}}{k !} \Delta\left(\log ^{k} m^{2}\right)=\sum_{k=0}^{\infty} \sum_{l=0}^{k} \frac{(-\epsilon)^{k}}{k !}\left(\begin{array}{c}
k \\
l
\end{array}\right) \log ^{k-l} m^{2} \otimes \log ^{l} m^{2} \\
& =\sum_{k, l=0}^{\infty} \frac{(-\epsilon)^{k+l}}{k ! l !} \log ^{k} m^{2} \otimes \log ^{l} m^{2}=m^{-2 \epsilon} \otimes m^{-2 \epsilon},
\end{aligned}
$$

where we have used eq. (2.3) in the second line. The formula for the coaction of the gamma function can be proven in the same way using the well-known formula

$$
e^{\gamma_{E} \epsilon} \Gamma(1+\epsilon)=\exp \sum_{k \geq 2} \frac{(-\epsilon)^{k} \zeta_{k}}{k},
$$

together with eqs. (2.4) and (2.5).

This example shows that in the case of the tadpole integral, it is possible to 'resum' the $\epsilon$-expansion of the coaction to obtain a version of the coaction valid to all orders in $\epsilon$. 
We note that, for these simple functions, this procedure can in fact be given a rigorous mathematical grounding in terms of the motivic coaction on multiple-zeta values and on the logarithm [42]. In more general cases, however, the functional dependence of the integral on the kinematic variables before expansion in $\epsilon$ is much more complicated and often involves functions of hypergeometric type.

The simplest nontrivial hypergeometric function is Gauss' ${ }_{2} F_{1}$ function. This function admits an Euler-type integral representation of the form

$$
{ }_{2} F_{1}(\alpha, \beta ; \gamma ; x)=\frac{\Gamma(\gamma)}{\Gamma(\alpha) \Gamma(\gamma-\alpha)} \int_{0}^{1} d u u^{\alpha-1}(1-u)^{\gamma-\alpha-1}(1-u x)^{-\beta}
$$

provided that the integral converges. Here we focus on a subset of cases, namely those where $\alpha, \beta, \gamma$ have the form $m+a \epsilon$, where $m \in \mathbb{Z}$. Under these restrictions, its Laurent expansion in $\epsilon$ involves MPLs as coefficients (which can be computed in an algorithmic way, cf. refs. [43-48]), and we can act with $\Delta$ on the coefficients order by order in the expansion. It is tantalizing to speculate whether it is possible to 'resum' the Laurent expansion after acting with the coaction. The central proposal of this paper is that, remarkably, this is indeed possible. Before we dive into the mathematical formalism in the next section, let us illustrate this result on a special case of a ${ }_{2} F_{1}$ function for which the Laurent coefficients can be written in closed form [49],

$$
{ }_{2} F_{1}(-\epsilon, 1 ; 1-\epsilon ; x)=1-\sum_{n=1}^{\infty} \epsilon^{n} \operatorname{Li}_{n}(x)=1-F(\epsilon, x) .
$$

Using eq. (2.3) for the coaction of the polylogarithms, we easily obtain

$$
\Delta[F(\epsilon, x)]=1 \otimes F(\epsilon, x)+F(\epsilon, x) \otimes x^{\epsilon},
$$

where we the coaction $\Delta$ acts order by order in the $\epsilon$ expansion.

In the remainder of this paper we argue that formulas like eq. (2.9) and (2.14) are not the exception but rather the rule, at least for very large classes of hypergeometric functions whose Laurent expansion can be expressed in terms of MPLs. More precisely, we argue that, for certain classes of functions, we can define a coaction $\Delta_{\epsilon}$ valid to all orders in the $\epsilon$ expansion. In our examples, the form of this coaction is obtained by replacing $\Delta$ by $\Delta_{\epsilon}$ everywhere in eq. (2.9) and (2.14). The Laurent expansion around $\epsilon=0$ then 'intertwines' the two coactions, i.e., if $L_{\epsilon}$ denotes the operator which assigns to a function its Laurent expansion, we have

$$
\left(L_{\epsilon} \otimes L_{\epsilon}\right) \Delta_{\epsilon}=\Delta L_{\epsilon},
$$

where in the right-hand side $\Delta$ acts order-by-order in the Laurent expansion.

\section{Positive geometries and canonical forms}

In this section we define the class of integrals that we will address in this paper, introduce some of the mathematical background and establish our notation. We will use the example of Euler's beta function to illustrate the different objects we introduce. 


\subsection{A class of integrals}

Let us consider an integral obtained by integrating a differential form $\omega$ over some domain $\gamma$. We start by characterizing the classes of integrands that will be of relevance in this paper. Our integrand $\omega$ depends on $n$ integration variables $u_{i}, i=1, \ldots, n$ as well as external variables $x_{j}, j=1, \ldots, m$ which we do not write explicitly. More precisely, $\omega$ is an $n$-form

$$
\omega=d \mathbf{u} \prod_{I} P_{I}(\mathbf{u})^{\alpha_{I}}
$$

with $d \mathbf{u}=d u_{1} \wedge \ldots \wedge d u_{n}$, where the $P_{I}$ are polynomials in the $u_{i}$ and $x_{j}$, and with $\alpha_{I} \in \mathbb{C}$. We assume the $P_{I}(\mathbf{u})$ to be irreducible over rational functions of the $x_{j}$. Furthermore, we assume that the exponents take the form $\alpha_{I}=n_{I}+a_{I} \epsilon$, with $n_{I} \in \mathbb{Z}, a_{I} \epsilon \in \mathbb{C}^{*}, \sum_{I} a_{I} \neq 0$, and where $\epsilon$ can be taken to be infinitesimally small. Finally, we define the decomposition $\omega=\Phi \varphi$ where

$$
\Phi=\prod_{I} P_{I}(\mathbf{u})^{a_{I} \epsilon} \quad \text { and } \quad \varphi=d \mathbf{u} \prod_{I} P_{I}(\mathbf{u})^{n_{I}} .
$$

We will always further restrict the form of these polynomials such that the coefficients in the Laurent expansion of the integrals $\int_{\gamma} \omega$ in $\epsilon$ only involve MPLs. We will be more specific about the form of the polynomials in subsequent sections.

The integration contour $\gamma$ is a $n$-dimensional cycle in

$$
X(\mathbb{C})=\mathbb{P}^{n}(\mathbb{C}) \backslash \bigcup_{I}\left\{P_{I}(\mathbf{u})=0\right\},
$$

where $\mathbb{P}^{n}(\mathbb{C})$ is the $n$-dimensional complex projective space. ${ }^{2}$ In other words, $\gamma$ is a domain with boundary contained in the union of the varieties defined by $P_{I}(\mathbf{u})=0$. Since $a_{I} \neq 0$, then $\Phi$ vanishes on the boundary of $\gamma$, at least for some ranges of values of $\epsilon$, and thus for all values by analytic continuation. As a consequence, there are no boundary contributions when performing integration by parts.

The natural mathematical framework to discuss such integrals is that of twisted homology and cohomology [27] — see also refs. [28, 30-32]. We define the twist $d \log \Phi$ (we will often call $\Phi$ the twist) and consider the covariant differential

$$
\nabla_{\Phi}=d+d \log \Phi \wedge
$$

We then have $d(\Phi \xi)=\Phi \nabla_{\Phi} \xi$, where $\xi$ can be any smooth differential form. Stokes' theorem implies that for an arbitrary smooth $(n-1)$-form $\xi$ we have

$$
\int_{\gamma} \Phi \varphi=\int_{\gamma} \Phi\left(\varphi+\nabla_{\Phi} \xi\right)
$$

As a consequence, the integrand is only defined up to adding a total covariant derivative, and we are therefore interested in the (twisted) cohomology groups

$$
H^{n}\left(X, \nabla_{\Phi}\right)=\left\{\varphi \mid \nabla_{\Phi} \varphi=0\right\} /\left\{\nabla_{\Phi} \xi\right\} .
$$

\footnotetext{
${ }^{2}$ Strictly speaking, $\left\{P_{I}(\mathbf{u})=P_{I}\left(u_{1}, \ldots, u_{n}\right)=0\right\}$ is an affine variety in $\mathbb{C}^{n}$. We use the same notation for the affine variety and its lift to projective space.
} 
Similarly, we can construct twisted homology groups by considering twisted cycles in $X(\mathbb{C})[27]$. The precise definition of the twisted cycles is not important for the rest of the paper. Here it suffices to say that they can be thought of as ordinary cycles $\gamma$, together with information on the Riemann sheet (with respect to the multi-valued function $\Phi$ ) on which this cycle is considered.

The cohomology groups in eq. (3.6) will play a prominent role in this paper. We will be particularly interested in determining explicit bases for the cohomology group associated to the integral we want to consider, which is in general an extremely difficult problem to solve. In some examples, however, it is easy to determine at least the dimension of these groups. One can start by counting the critical points of the function $\Phi$, i.e. the number of independent solutions to the equation

$$
d \log \Phi=0,
$$

which gives an upper bound for the dimensionality. This upper bound is saturated under certain conditions outlined for example in ref. [27], see also refs. [31, 50, 51]. In particular, the bound is saturated in each of the examples studied in this paper. For a discussion of the limitations of this approach in the context of Feynman integrals, see e.g. ref. [50].

\subsection{Integrals and positive geometries}

As already mentioned, it can be very difficult to construct explicit bases for the cohomology groups. Here, however, we are not interested in the most general case, but we restrict our attention to those cases where they are generated by (wedge products of) $d \log$-forms, i.e., by differential forms with logarithmic singularities along the varieties $\left\{P_{I}(\mathbf{u})=0\right\}$.

A particularly convenient such geometric setting is that of positive geometries [26], whose definition we briefly recall in this section. Let $Y(\mathbb{C})$ be an irreducible complex projective variety of dimension $n$, corresponding to the solution in $\mathbb{P}^{n}(\mathbb{C})$ of homogeneous polynomial equations with rational coefficients. We assume that $Y(\mathbb{C})$ has no nonzero holomorphic $n$-forms. We denote by $\mathbb{P}^{n}(\mathbb{R})$ the $n$-dimensional real projective space, and by $Y(\mathbb{R})$ the real part of $Y(\mathbb{C})$, i.e., the solution of the same polynomial equations in $\mathbb{P}^{n}(\mathbb{R})$. For concreteness, we will always be working in an affine chart of projective space, with affine coordinates $\mathbf{u}=\left(u_{1}, \ldots, u_{n}\right)$. In this chart, the surfaces are described by the polynomial equations $P_{I}(\mathbf{u})=0$, which carve out $n$-dimensional cells $\Gamma_{j}$ in $Y(\mathbb{R})$. In the following we will always be interested in the case where $Y(\mathbb{C})=\mathbb{P}^{n}(\mathbb{C})$. A positive geometry is a pair $\left(\mathbb{P}^{n}(\mathbb{C}), \Gamma_{j}\right)$ together with a differential form $\Omega\left(\mathbb{P}^{n}(\mathbb{C}), \Gamma_{j}\right)$, called the canonical form, with simple poles on the boundary of $\Gamma_{j}$. The form $\Omega\left(\mathbb{P}^{n}(\mathbb{C}), \Gamma_{j}\right)$ is unique (up to normalization) because we assume that there are no nonzero holomorphic $n$-forms. We also require that all boundary components are themselves positive geometries whose canonical form is given by ${ }^{3}$

$$
\Omega\left(\mathbb{P}^{n-1}(\mathbb{C}), \partial_{I} \Gamma_{j}\right)=\operatorname{Res}_{\partial_{I} \Gamma_{j}} \Omega\left(\mathbb{P}^{n}(\mathbb{C}), \Gamma_{j}\right) .
$$

We refer the reader to ref. [26] for a more precise definition.

\footnotetext{
${ }^{3}$ To be more precise, recall that we have assumed the $P_{I}(\mathbf{u})$ to be irreducible polynomials. Then the boundary component $\partial_{I} \Gamma_{j}$ of $\Gamma_{j}$ is the part of the boundary of $\Gamma_{j}$ that lies in the variety $\left\{P_{I}(\mathbf{u})=0\right\}$. The $\partial_{I} \Gamma_{j}$ are themselves positive geometries $\left(\mathbb{P}^{n-1}(\mathbb{C}), \partial_{I} \Gamma_{j}\right)$ with associated canonical forms.
} 
For $n=0$, the $\Gamma_{j}$ are points and $\Omega\left(\mathbb{P}^{0}(\mathbb{C}), \Gamma_{j}\right)= \pm 1$ for any $j$. For $n>0$, we can choose the forms $\Omega\left(\mathbb{P}^{n}(\mathbb{C}), \Gamma_{j}\right)$ to be $d \log$-forms. In the following, we will refer to the cells $\Gamma_{j}$ themselves as positive geometries, and we will denote their canonical forms simply by $\Omega\left(\Gamma_{j}\right)$. We note that the map $\Omega$, which associates to a positive geometry $\Gamma_{j}$ its canonical form, is closely related to the map $c_{0}$ that has appeared in ref. [52].

For the integrals considered in this paper, the $\Gamma_{j}$ and the associated canonical forms $\Omega\left(\Gamma_{j}\right)$ are natural candidates for the generators of the (co)homology groups associated to the geometry underlying the integral under consideration. In other words, we will be considering integrals of the form

$$
\int_{\Gamma_{j^{\prime}}} \Phi \Omega\left(\Gamma_{j}\right)
$$

Integrals of this type, where we integrate the canonical form of the positive geometry $\Gamma_{j}$ over some other positive geometry $\Gamma_{j^{\prime}}$ were called canonical integrals in ref. [26]. Here we slightly generalize this notion and consider canonical integrals with a twist $d \log \Phi[28,30]$. For generic $a_{I}$ in $\Phi$, the integral above is well-defined even if $j=j^{\prime}$, as long as it converges. The result can then be analytically continued to any $a_{I} \in \mathbb{C}$ (up to poles).

In general, not all canonical integrals are independent and it is convenient to find bases $\vec{\gamma} \equiv\left(\gamma_{1}, \ldots, \gamma_{k}\right)$ of the associated homology group and $\vec{\varphi} \equiv\left(\varphi_{1}, \ldots, \varphi_{k}\right)$ of the associated cohomology group. The elements of the cohomology group are equivalence classes of differential forms, and since we restrict ourselves to canonical integrals, each class contains a $d \log$ representative. Throughout this paper we simply represent each class by this $d \log$ form, keeping in mind that the elements of the cohomology groups are actually equivalence classes and not differential forms. Similarly, we will represent elements of the homology groups simply by the cycles $\Gamma_{j}$.

As already stated at the end of the previous section, the dimension $k$ of the homology and cohomology groups can be determined by counting the critical points of $\Phi$. Any cell $\Gamma_{j}$ can then be written as a linear combination of the basis elements $\gamma_{i}$, and similarly for the associated canonical forms and the basis $\varphi_{i}$. It is known how to find such bases in the case where the $P_{I}(\mathbf{u})$ are either all linear (an "arrangement of hyperplanes") or all linear but one, where the latter can have degree up to $n[26,30]$. In practice, this is sufficient for all the examples we will be interested in this paper. For instance, the homology group of intersections of hyperplanes in general position ${ }^{4}$ is generated by bounded chambers (the cells that do not extend to infinity) [27]. A cycle $\Gamma$ defined by having its boundaries on hyperplanes is a positive geometry whose canonical form can be written down explicitly as follows. If the hyperplanes at the boundaries are defined by the linear equations $P_{1}(\mathbf{u})=\ldots=P_{k}(\mathbf{u})=0$, then the associated canonical form is uniquely determined up to a sign to be

$$
\Omega(\Gamma)=d \log \frac{P_{2}(\mathbf{u})}{P_{1}(\mathbf{u})} \wedge \ldots \wedge d \log \frac{P_{k}(\mathbf{u})}{P_{k-1}(\mathbf{u})}
$$

\footnotetext{
${ }^{4}$ When the hyperplanes are not in general position there may be non-normal crossings, in which case we need to perform a blow-up around the non-normal crossing surface or, using physicists' nomenclature, use sector decomposition to resolve the overlapping singularities.
} 
As an example, consider the case where $n=1$, i.e., there is a single integration variable $u$. The solutions to $\prod_{I} P_{I}(u)=0$ are points $u_{i}$, which are a special case of hyperplanes in $\mathbb{P}^{1}(\mathbb{R})$. The bounded chambers are the intervals between consecutive finite solutions, but any interval $\left[u_{i}, u_{j}\right]$ can be seen as a positive geometry with the canonical form

$$
\Omega\left(\left[u_{i}, u_{j}\right]\right)=d \log \frac{u-u_{i}}{u-u_{j}} .
$$

The boundary components of $\left[u_{i}, u_{j}\right]$ are the points $u_{i}$ and $u_{j}$, which are 0-dimensional positive geometries with the canonical forms

$$
\underset{u=u_{i}}{\operatorname{Res}} \Omega\left(\left[u_{i}, u_{j}\right]\right)=\Omega\left(\left\{u_{i}\right\}\right)=1, \quad \operatorname{Res}_{u=u_{j}} \Omega\left(\left[u_{i}, u_{j}\right]\right)=\Omega\left(\left\{u_{j}\right\}\right)=-1,
$$

in agreement with the recursive construction of eq. (3.8). We can also consider cycles extending to infinity, which are also positive geometries whose canonical forms are given by

$$
\Omega\left(\left[u_{i}, \infty\right)\right)=d \log \left(u-u_{i}\right) .
$$

Example: Euler's beta function. Consider the integral

$$
\int_{\gamma} \omega=\int_{\gamma} u^{\alpha_{1}}(1-u)^{\alpha_{2}} d u
$$

This integral contains as a special case Euler's integral representation of the beta function,

$$
B(\alpha, \beta)=\int_{0}^{1} u^{\alpha-1}(1-u)^{\beta-1} d u,
$$

which converges for $\mathfrak{R e}(\alpha)>0$ and $\mathfrak{R e}(\beta)>0$. The integrand $\omega$ has two linear factors, $P_{1}(u)=u$ and $P_{2}(u)=1-u$, raised to the exponents $\alpha_{1}=n_{1}+a_{1} \epsilon$ and, $\alpha_{2}=n_{2}+a_{2} \epsilon$. The multivalued function $\Phi$ is $\Phi=u^{a_{1} \epsilon}(1-u)^{a_{2} \epsilon}$.

There is a single bounded chamber, $[0,1]$, defined by the polynomials $P_{1}(u)$ and $P_{2}(u)$. This is consistent with the fact that there is a single solution to $d \log \Phi=0$, i.e, $\Phi$ has a single critical point. The associated canonical form is

$$
\Omega([0,1])=d \log \frac{u}{u-1} .
$$

Note that there are other positive geometries we could have considered (another example is $[1, \infty])$, but since $P_{1}(u)$ and $P_{2}(u)$ are linear in $u$ it is sufficient to consider the bounded chamber. Furthermore, through integration-by-parts relations, the one-form in eq. (3.15) gives a basis of the first cohomology group of eq. (3.13). In other words, any integral of the type (3.13) is a multiple of

$$
\int_{0}^{1} d u u^{a_{1} \epsilon}(1-u)^{a_{2} \epsilon}\left(\frac{1}{u}-\frac{1}{u-1}\right) .
$$

This is straightforward to check by explicit calculation, using well-known identities among gamma functions. 


\subsection{Pairings of cycles and forms}

Having discussed how to construct the twisted homology and cohomology groups associated with a canonical integral, we now discuss how to pair elements of these groups through complex-valued bilinear maps.

We denote by $\vec{\gamma}$ a basis of the homology group and by $\vec{\varphi}$ a basis of the cohomology group. The most natural pairing is to associate cycles $\gamma_{k} \in \vec{\gamma}$ with forms $\varphi_{l} \in \vec{\varphi}$ to compute the so-called period matrix

$$
P_{k l}(\vec{\gamma} ; \vec{\varphi} ; \Phi)=\int_{\gamma_{k}} \Phi \varphi_{l},
$$

where each row corresponds to a cycle and each column corresponds to a form. The matrix $P$ is a square matrix whose dimension is given by the dimension of the (co)homology group, which we recall can be determined by counting the critical points of $\Phi$. For a given twist $\Phi$, cycle $\gamma$ and integrand $\omega=\Phi \varphi$, and assuming that $\gamma$ is in the associated homology group and $\varphi$ is in the associated cohomology group, any integral $\int_{\gamma} \omega$ can be written as a linear combination of the elements of the period matrix,

$$
\int_{\gamma} \omega=\sum_{k, l} c_{k l} P_{k l}(\vec{\gamma} ; \vec{\varphi} ; \Phi)
$$

The algebraic properties of any integral of this type can then be studied from the entries of the period matrix.

A less obvious pairing we can construct is between two differential forms. Let us assume we have two bases $\vec{\varphi}$ and $\vec{\psi}$, not necessarily distinct, of the same twisted cohomology group. We can then compute intersection numbers $\left\langle\varphi_{i}, \psi_{j}\right\rangle_{\Phi}$ between these forms. To be more precise, we must first construct a dual twisted cohomology group, which is also generated by $\vec{\psi}$ but for which the covariant differential is $\nabla_{\Phi^{-1}}$. In our case, this corresponds to taking $\epsilon \rightarrow-\epsilon$ in $\Phi$. We can then pair generators $\left\langle\varphi_{i}\right|$ of the cohomology with elements $\left|\psi_{j}\right\rangle$ of the dual cohomology [27] (see also ref. [28]),

$$
\left\langle\varphi_{i}, \psi_{j}\right\rangle_{\Phi}=\frac{1}{(2 \pi i)^{2}} \int_{X(\mathbb{C})} \iota_{\Phi}\left(\varphi_{i}\right) \wedge \psi_{j}
$$

with $X(\mathbb{C})$ as defined in eq. (3.3) and $\iota_{\Phi}$ the map that associates to a form $\varphi_{i}$ a form $\iota_{\Phi}\left(\varphi_{i}\right)$ in the same cohomology class but with compact support so that the integral is well defined $[28,53]$. Intersection numbers can then be arranged in the matrix

$$
C_{k l}(\vec{\varphi} ; \vec{\psi} ; \Phi)=\left\langle\varphi_{k}, \psi_{l}\right\rangle_{\Phi},
$$

which has the same dimensions as the period matrix $P$.

The matrix of intersection numbers $C(\vec{\varphi} ; \vec{\psi} ; \Phi)$ in eq. (3.20) will play a very important role in the construction of our coaction. Therefore we need efficient ways of computing intersection numbers. The definition of the intersection numbers in eq. (3.19) is not the most convenient for practical calculations, so we now discuss three alternative ways to compute them in the cases we are interested in. In the case where $n=1$ and the $\varphi_{i}$ and 
$\psi_{j}$ are $d \log$-forms, which covers several of the examples of this paper, a more explicit way to compute the intersection numbers is $[28,30]$

$$
\left\langle\varphi_{i}, \psi_{j}\right\rangle_{\Phi}=\sum_{u_{p} \in \mathcal{P}(\Phi)} \frac{\operatorname{Res}_{u=u_{p}} \varphi_{i} \operatorname{Res}_{u=u_{p}} \psi_{j}}{\operatorname{Res}_{u=u_{p}} d \log \Phi}
$$

where $\mathcal{P}(\Phi)$ is the set of poles of $d \log \Phi$. This very explicit formula can be generalized to the case where $n>1$ [32].

An alternative formula was proposed in ref. [28]. For $n=1$ and setting $u_{1}=u$,

$$
\left\langle\varphi_{i}, \psi_{j}\right\rangle_{\Phi}=\left.\sum_{u^{*}}\left(\frac{\partial^{2} \log \Phi}{\partial u^{2}}\right)^{-1} \widehat{\varphi}_{i} \widehat{\psi}_{j}\right|_{u=u^{*}}
$$

where the sum is over the critical points, i.e., the points $u^{*}$ satisfying $d \log \Phi\left(u^{*}\right)=0$, and $\varphi_{i}=\widehat{\varphi}_{i} d u$ and similarly for $\psi_{j}$. In the case $n=2$, with $\left(u_{1}, u_{2}\right)=(u, v)$,

$$
\left\langle\varphi_{i}, \psi_{j}\right\rangle_{\Phi}=\left.\sum_{\left(u^{*}, v^{*}\right)} \operatorname{det}^{-1}\left(\begin{array}{ll}
\frac{\partial^{2} \log \Phi}{\partial u^{2}} & \frac{\partial^{2} \log \Phi}{\partial u \partial v} \\
\frac{\partial^{2} \log \Phi}{\partial u \partial v} & \frac{\partial^{2} \log \Phi}{\partial v^{2}}
\end{array}\right) \widehat{\varphi}_{i} \widehat{\psi}_{j}\right|_{(u, v)=\left(u^{*}, v^{*}\right)},
$$

where the sum extends over the critical points $\left(u^{*}, v^{*}\right)$ satisfying

$$
\partial_{u} \log \Phi\left(u^{*}, v^{*}\right)=\partial_{v} \log \Phi\left(u^{*}, v^{*}\right)=0 .
$$

Finally, we can also use the period matrix to compute a certain matrix of intersection numbers. Let $P(\vec{\gamma} ; \vec{\varphi} ; \Phi)$ be the period matrix constructed from the contours $\vec{\gamma}$ and $d \log$ forms $\vec{\varphi}$ as defined above. Then, the matrix $C(\Omega(\vec{\gamma}) ; \vec{\varphi} ; \Phi)$ is related to the period matrix $P(\vec{\gamma} ; \vec{\varphi} ; \Phi)$ through [30]

$$
P(\vec{\gamma} ; \vec{\varphi} ; \Phi)=C(\Omega(\vec{\gamma}) ; \vec{\varphi} ; \Phi)(1+\mathcal{O}(\epsilon))
$$

where we defined $\Omega(\vec{\gamma}) \equiv\left(\Omega\left(\gamma_{1}\right), \ldots, \Omega\left(\gamma_{k}\right)\right)$. We note that this relation is in agreement with the fact that the entries of $P(\vec{\gamma} ; \vec{\varphi} ; \Phi)$ are multivalued functions while those of $C(\Omega(\vec{\gamma}) ; \vec{\varphi} ; \Phi)$ are not. Indeed, given our choice of using a basis of $d \log$ forms, the leading order in the Laurent expansion of $P(\vec{\gamma} ; \vec{\varphi} ; \Phi)$ is single-valued.

Example: Euler's beta function. We return to the example of eq. (3.13). In the previous section we established that it is sufficient to study the beta function in eq. (3.14). Building on the discussion on positive geometries, we choose the contour $\gamma=[0,1]$ as the generator of the homology group, and the associated canonical form is

$$
\Omega(\gamma)=\left(\frac{1}{u}+\frac{1}{1-u}\right) d u
$$

In the terminology of this section, this means we have a one-dimensional period matrix

$$
P(\gamma ; \Omega(\gamma) ; \Phi)=\frac{a_{1}+a_{2}}{a_{1} a_{2} \epsilon} \frac{\Gamma\left(1+a_{1} \epsilon\right) \Gamma\left(1+a_{2} \epsilon\right)}{\Gamma\left(1+\left(a_{1}+a_{2}\right) \epsilon\right)}=\frac{a_{1}+a_{2}}{a_{1} a_{2} \epsilon}+\mathcal{O}\left(\epsilon^{0}\right)
$$


We will frequently choose bases of the cohomology group that are not the canonical forms of our chosen generators of the homology group. Let us therefore expand this illustrative example by considering a different generator of the cohomology group,

$$
\varphi=\frac{d u}{1-u},
$$

which happens to be the canonical form of a cycle extending from $u=1$ to infinity.

Keeping the same generator of the homology group, we get

$$
P(\gamma ; \varphi ; \Phi)=\frac{1}{a_{2} \epsilon} \frac{\Gamma\left(1+a_{1} \epsilon\right) \Gamma\left(1+a_{2} \epsilon\right)}{\Gamma\left(1+\left(a_{1}+a_{2}\right) \epsilon\right)}=\frac{1}{a_{2} \epsilon}+\mathcal{O}\left(\epsilon^{0}\right) .
$$

Clearly, the two choices are dependent,

$$
P(\gamma ; \Omega(\gamma) ; \Phi)=\frac{a_{1}+a_{2}}{a_{1}} P(\gamma ; \varphi ; \Phi) .
$$

Let us now compute the intersection of the two choices of forms. We can use eq. (3.21), which requires the following residues:

$$
\begin{aligned}
& \operatorname{Res}_{u=0} \varphi=0, \quad \operatorname{Res}_{u=1} \varphi=-1, \quad \operatorname{Res}_{u=\infty} \varphi=1, \\
& \operatorname{Res}_{u=0} \Omega(\gamma)=1, \quad \operatorname{Res}_{u=1} \Omega(\gamma)=-1, \quad \operatorname{Res}_{u=\infty} \Omega(\gamma)=0,
\end{aligned}
$$

and, given that $\log \Phi=a_{1} \epsilon \log (u)+a_{2} \epsilon \log (1-u)$,

$$
\operatorname{Res}_{u=0} d \log \Phi=a_{1} \epsilon, \quad \operatorname{Res}_{u=1} d \log \Phi=a_{2} \epsilon, \quad \operatorname{Res}_{u=\infty} d \log \Phi=-\left(a_{1}+a_{2}\right) \epsilon .
$$

Then we find that, for instance,

$$
C(\Omega(\gamma) ; \Omega(\gamma) ; \Phi)=\frac{a_{1}+a_{2}}{a_{1} a_{2} \epsilon} \quad \text { and } \quad C(\Omega(\gamma) ; \varphi ; \Phi)=\frac{1}{a_{2} \epsilon} .
$$

We take this opportunity to observe that the poles in $\epsilon$ of the period matrix arise from logarithmic singularities at the boundary of the integration region, which are regulated by $\epsilon$. The matrix of intersection numbers captures the same information (see, e.g., ref. [28]). For $\gamma=[0,1]$ and when using the form $\varphi$ there is only a contribution from the boundary of $\gamma$ at $u=1$, where $\varphi$ also has a pole. When using the canonical form $\Omega(\gamma)$ there is also a contribution from $u=0$.

\section{A coaction on integrals}

Having established our notation in the previous sections, we now present the main result of this paper, which is a coaction $\Delta_{\epsilon}$ acting on canonical integrals with a twist as defined in the previous section.

The coaction is given by

$$
\Delta_{\epsilon} \int_{\gamma} \omega=\sum_{i, j}\left[C^{-1}(\Omega(\vec{\gamma}) ; \vec{\varphi} ; \Phi)\right]_{i j} \int_{\gamma} \Phi \varphi_{i} \otimes \int_{\gamma_{j}} \omega,
$$


where $\omega=\Phi \varphi, \vec{\varphi} \equiv\left\{\varphi_{1}, \ldots, \varphi_{k}\right\}$ is a set of differential forms that generate the cohomology group for the geometry associated to our family of integrals, and $\vec{\gamma} \equiv\left\{\gamma_{1}, \ldots, \gamma_{k}\right\}$ is a set of cycles that generate the corresponding homology group. The integrands $\varphi$ and cycles $\gamma$ are elements of the cohomology and homology groups generated by $\vec{\varphi}$ and $\vec{\gamma}$. We stress that the twist $\Phi$ is common to all components in eq. (4.1). Furthermore, this coaction is only valid in the case where the homology group is generated by cells $\gamma_{i}$, such that for each cell there exists a unique ${ }^{5}$ differential form (up to normalization) with logarithmic singularities on the boundary of $\gamma_{i}$. In this way the matrix of intersection numbers $C(\Omega(\vec{\gamma}) ; \vec{\varphi} ; \Phi)$, a priori only defined from differential forms, knows about the choice of contours $\vec{\gamma}$. For example, we may consider that each $\gamma_{i}$ is a positive geometry with canonical form $\Omega\left(\gamma_{i}\right)$.

We conjecture that eq. (4.1) satisfies a highly nontrivial relation already stated in eq. (2.15), namely that it is related to the coaction on MPLs,

$$
\left(L_{\epsilon} \otimes L_{\epsilon}\right) \Delta_{\epsilon}=\Delta L_{\epsilon},
$$

where $L_{\epsilon}$ is the operator which assigns to a function its Laurent expansion around $\epsilon=0$. Before verifying this conjecture in a series of examples in the following sections, we first make some comments on eq. (4.1).

First, as argued previously, several algebraic properties of a generic integral are determined by its associated period matrix, as defined in eq. (3.17). It is thus particularly interesting to study the coaction of the entries of the period matrix (the coaction of a generic integral then follows from the relation in eq. (3.18)). Consider eq. (4.1) in the special case where $\gamma=\gamma_{k}$ and $\varphi=\varphi_{l}$. It then follows that the coaction on the period matrix is simply obtained by matrix multiplication:

$$
\Delta_{\epsilon} P_{k l}(\vec{\gamma} ; \vec{\varphi} ; \Phi)=\sum_{i, j}\left[C^{-1}(\Omega(\vec{\gamma}) ; \vec{\varphi} ; \Phi)\right]_{i j} P_{k i}(\vec{\gamma} ; \vec{\varphi} ; \Phi) \otimes P_{j l}(\vec{\gamma} ; \vec{\varphi} ; \Phi) .
$$

Second, one can choose generators of the (co)homology such that $C(\Omega(\vec{\gamma}) ; \vec{\varphi} ; \Phi)=\delta_{i j}$, in which case the coaction takes a particularly simple form:

$$
\Delta_{\epsilon} P_{k l}(\vec{\gamma} ; \vec{\varphi} ; \Phi)=\sum_{i} P_{k i}(\vec{\gamma} ; \vec{\varphi} ; \Phi) \otimes P_{i l}(\vec{\gamma} ; \vec{\varphi} ; \Phi) .
$$

Finally, we comment on the relation between the coaction proposed here, eq. (4.1), and the one proposed in refs. $[9,10]$. The latter relies on diagonalizing the generators of the (co)homology group using the semi-simple projection of the period matrix instead of the matrix of intersection numbers. For all examples given in this paper the two procedures give the same result because of eq. (3.24). In this paper we prefer the formulation of eq. (4.1) because it is more manifestly symmetric in its treatment of the basis of contours and differential forms. We also note that our conjecture in eq. (4.1) is very reminiscent of the formula for the coaction of the tree-level string amplitudes [54-56], with the inverse matrix of intersection numbers being identified with the KLT kernel [57] and $\epsilon$ related to the string tension $\alpha^{\prime}$.

\footnotetext{
${ }^{5}$ As a counterexample, in the case where the algebraic variety $Y(\mathbb{C})$ discussed in section 3 admits nonzero holomorphic forms, there is no unique assignment of forms to contours. This situation is ruled out when working with positive geometries.
} 
Example: Euler's beta function. We return a last time to the example of Euler's beta function as an illustration of the application of our coaction formula. We recall that

$$
\int_{0}^{1} d u u^{\alpha_{1}}(1-u)^{\alpha_{2}}=\frac{\Gamma\left(1+\alpha_{1}\right) \Gamma\left(1+\alpha_{2}\right)}{\Gamma\left(2+\alpha_{1}+\alpha_{2}\right)}
$$

where in our case $\alpha_{i}=n_{i}+a_{i} \epsilon$, with $n_{i} \in \mathbb{Z}$. We take the generator of the cohomology group to be the canonical form constructed from the integration contour, see eq. (3.15). Using eqs. (3.26) and (3.32) we find that:

$$
\Delta_{\epsilon}\left[\frac{\Gamma\left(1+\alpha_{1}\right) \Gamma\left(1+\alpha_{2}\right)}{\Gamma\left(2+\alpha_{1}+\alpha_{2}\right)}\right]=\frac{\Gamma\left(1+a_{1} \epsilon\right) \Gamma\left(1+a_{2} \epsilon\right)}{\Gamma\left(1+\left(a_{1}+a_{2}\right) \epsilon\right)} \otimes \frac{\Gamma\left(1+\alpha_{1}\right) \Gamma\left(1+\alpha_{2}\right)}{\Gamma\left(2+\alpha_{1}+\alpha_{2}\right)} .
$$

Exactly the same result is obtained using the alternative generator of the cohomology group in eq. (3.27). It is straightforward to check that eq. (4.2) holds to an arbitrary order in the Laurent expansion around $\epsilon=0$.

We can write eq. (4.6) explicitly in terms of Euler's beta function. If we let $\alpha=n_{a}+a \epsilon$, $\beta=n_{b}+b \epsilon$ in eq. (3.14), with $n_{a}, n_{b} \in \mathbb{Z}$, then

$$
\Delta_{\epsilon}(B(\alpha, \beta))=\frac{(a \epsilon)(b \epsilon)}{(a+b) \epsilon} B(a \epsilon, b \epsilon) \otimes B(\alpha, \beta) .
$$

Finally, we note that eq. (4.6) is consistent with the coaction given in eq. (2.9) for the gamma function: upon using the fact that the coaction of a product of two functions is the product of the coaction of the functions, $\Delta(f \cdot g)=\Delta(f) \cdot \Delta(g)$, and in particular, that eq. (2.9) also implies

$$
\Delta\left[\frac{e^{-\gamma_{E} \epsilon}}{\Gamma(1+\epsilon)}\right]=\left[\frac{e^{-\gamma_{E} \epsilon}}{\Gamma(1+\epsilon)}\right] \otimes\left[\frac{e^{-\gamma_{E} \epsilon}}{\Gamma(1+\epsilon)}\right]
$$

one may readily verify the coaction in (4.6).

\section{Gauss' hypergeometric function ${ }_{2} F_{1}$}

In this section we discuss Gauss' hypergeometric function ${ }_{2} F_{1}$ in detail. We start from Euler's integral representation given in eq. (2.12) and restrict ourselves to the class of functions defined below it. Having already discussed the coaction on beta functions, it is sufficient to study the parametric integral

$$
\int_{0}^{1} u^{n_{0}+a_{0} \epsilon}(1-u)^{n_{1}+a_{1} \epsilon}(1-x u)^{n_{1 / x}+a_{1 / x} \epsilon} d u=\frac{\Gamma(\alpha) \Gamma(\gamma-\alpha)}{\Gamma(\gamma)}{ }_{2} F_{1}(\alpha, \beta ; \gamma ; x)
$$

where $\alpha=1+n_{0}+a_{0} \epsilon, \beta=-n_{1 / x}-a_{1 / x} \epsilon$, and $\gamma=2+n_{0}+n_{1}+\epsilon\left(a_{0}+a_{1}\right)$ and, in accordance with the framework established in section $3, a_{0}, a_{1}, a_{1 / x} \in \mathbb{C}^{*}$ and $n_{i} \in \mathbb{Z}$. It is well known that for fixed and generic $a_{0}, a_{1}$ and $a_{1 / x}$, the space spanned by the parametric integral above is two-dimensional (i.e., the cohomology group has dimension 2). The linear relations between the functions in this space - relations between integrals with different assignments of the integers $n_{i}$ - follow from Gauss' contiguous relations (see, e.g., ref. [58]), or equivalently from integration-by-parts identities of eq. (5.1). 


\subsection{The coaction in the basis of canonical forms}

The integral in eq. (5.1) falls into the class of canonical integrals with a twist defined in section 3.2. Following the notation introduced there, we write the integrand of eq. (5.1) as

$$
\omega=\Phi \varphi
$$

where

$$
\begin{aligned}
& \Phi=u^{a_{0} \epsilon}(1-u)^{a_{1} \epsilon}(1-x u)^{a_{1 / x} \epsilon}, \\
& \varphi=u^{n_{0}}(1-u)^{n_{1}}(1-x u)^{n_{1 / x}} d u .
\end{aligned}
$$

The three linear factors $P_{0}(u)=u, P_{1}(u)=1-u$ and $P_{1 / x}(u)=1-x u$ define the 0-dimensional hyperplanes

$$
H_{0}=\{u=0\}, \quad H_{1}=\{u=1\}, \quad H_{1 / x}=\{u=1 / x\},
$$

considered to be in general position (i.e., $x \neq 0,1, \infty$ ). The dimension of the (co)homology group is given by the number of critical points of $\log \Phi$, i.e., the number of solutions to $d \log \Phi=0$ in $\mathbb{C} \backslash\{0,1,1 / x\}$. It is straightforward to see that there are two solutions to this equation, in agreement with the dimension of the cohomology group deduced from Gauss' contiguous relations for the ${ }_{2} F_{1}$ function.

It is natural to consider the positive geometries defined by the straight-line segments

$$
\gamma_{1}=[0,1] \quad \text { and } \quad \gamma_{2}=[0,1 / x]
$$

and the associated canonical forms

$$
\psi_{1} \equiv \Omega\left(\gamma_{1}\right)=d \log \frac{u}{u-1}, \quad \psi_{2} \equiv \Omega\left(\gamma_{2}\right)=d \log \frac{u}{u-1 / x},
$$

as generators of the (co)homology groups of eq. (5.1).

For a generic integrand $\varphi$ as in eq. (5.3), we can write the integrals over $\gamma_{1}$ or $\gamma_{2}$ in terms of Gauss hypergeometric functions:

$$
\begin{aligned}
\int_{\gamma_{1}} \Phi \varphi & =\frac{\Gamma(\alpha) \Gamma(\gamma-\alpha)}{\Gamma(\gamma)}{ }_{2} F_{1}(\alpha, \beta ; \gamma ; x), \\
\int_{\gamma_{2}} \Phi \varphi & =x^{-\alpha} \frac{\Gamma(\alpha) \Gamma(1-\beta)}{\Gamma(1+\alpha-\beta)}{ }_{2} F_{1}\left(\alpha, 1+\alpha-\gamma ; 1+\alpha-\beta ; \frac{1}{x}\right),
\end{aligned}
$$

with $\alpha=1+n_{0}+a_{0} \epsilon, \beta=-n_{1 / x}-a_{1 / x} \epsilon$, and $\gamma=2+n_{0}+n_{1}+\epsilon\left(a_{0}+a_{1}\right)$. For concreteness we take $0<x<1$, but all results can easily be analytically continued to any value of $x$. We can then explicitly construct the period matrix

$$
P(\vec{\gamma} ; \vec{\psi} ; \Phi)=\left(\begin{array}{l}
\int_{\gamma_{1}} \Phi \psi_{1} \int_{\gamma_{1}} \Phi \psi_{2} \\
\int_{\gamma_{2}} \Phi \psi_{1} \int_{\gamma_{2}} \Phi \psi_{2}
\end{array}\right),
$$


with $\vec{\gamma} \equiv\left(\gamma_{1}, \gamma_{2}\right)$ and $\vec{\psi}=\left(\psi_{1}, \psi_{2}\right)$. We note that all the entries of the period matrix are canonical integrals with a twist, as defined in section 3.2.

To compute the coaction from eq. (4.3) we need the intersection matrix $C(\Omega(\vec{\gamma}) ; \vec{\psi} ; \Phi)$ which is easily computed from eq. (3.21):

$$
C(\Omega(\vec{\gamma}) ; \vec{\psi} ; \Phi)=C(\vec{\psi} ; \vec{\psi} ; \Phi)=\left(\begin{array}{cc}
\frac{1}{a_{0} \epsilon}+\frac{1}{a_{1} \epsilon} & \frac{1}{a_{0} \epsilon} \\
\frac{1}{a_{0} \epsilon} & \frac{1}{a_{0} \epsilon}+\frac{1}{a_{1 / x} \epsilon}
\end{array}\right) .
$$

By comparing the leading order of the Laurent expansion of the period matrix with the matrix above we can check that eq. (3.24) holds.

Having at our disposal the matrices $P$ and $C$, we can simply compute the coaction by inverting the matrix in eq. (5.8) and then using eq. (4.3). We have done this and checked that it satisfies eq. (4.2) by computing explicitly the first few terms in the Laurent expansion of all the entries of the period matrix in eq. (5.7), and then applying the coaction on the Laurent coefficients expressed in terms of MPLs (the last step was done using the Mathematica package PolyLogTools [59]).

\subsection{Coaction in an orthonormal basis}

The expression for the coaction obtained in the previous section is not particularly elegant, mainly because the matrix in eq. (5.8) (or more precisely, its inverse) is not so simple. Here, we show how to obtain a more elegant coaction by choosing a different basis of the cohomology group.

We find it convenient to choose basis elements $\varphi_{i}$ such that the matrix of intersection numbers $C(\vec{\varphi} ; \vec{\psi} ; \Phi)$ has a minimum number of nonvanishing off-diagonal elements. This principle is not necessarily compatible with the choice of using the canonical forms associated with positive geometries. Indeed, $C$ will be diagonal if each $\varphi_{i}$ is taken to be a $d \log$-form whose singularities overlap with the boundary components of $\gamma_{j}$ if and only if $i=j$. However, canonical forms have nonvanishing residues on all boundary components, which implies that overlaps occur between different basis elements. In the following we present a basis in which $C$ is diagonal. We stress nevertheless that this choice is only a matter of preference.

For the example at hand, we can choose a form $\tilde{\varphi}_{1}$ to be singular only at $H_{1}$, and a form $\tilde{\varphi}_{2}$ to be singular only at $H_{1 / x}$. For instance, keeping the original basis of cycles $\gamma_{1}$ and $\gamma_{2}$, we may consider the alternative basis of forms

$$
\tilde{\varphi}_{1}=-d \log (1-u)=\frac{d u}{1-u}, \quad \tilde{\varphi}_{2}=-d \log (1-x u)=x \frac{d u}{1-x u},
$$

which generate the same cohomology group as the $\psi_{1}=\Omega\left(\gamma_{1}\right)$ and $\psi_{2}=\Omega\left(\gamma_{2}\right)$ given in eq. (5.5). With $\tilde{\varphi}=\left(\tilde{\varphi}_{1}, \tilde{\varphi}_{2}\right)$, we find

$$
C(\vec{\psi} ; \tilde{\varphi} ; \Phi)=\left(\begin{array}{cc}
\frac{1}{a_{1} \epsilon} & 0 \\
0 & \frac{1}{a_{1 / x} \epsilon}
\end{array}\right)
$$


It is then clear that $\vec{\varphi}=\left(\varphi_{1}, \varphi_{2}\right)$ with

$$
\varphi_{1}=a_{1} \epsilon \frac{d u}{1-u}, \quad \varphi_{2}=a_{1 / x} \epsilon x \frac{d u}{1-x u},
$$

produces a matrix of intersection numbers that is unity, $C(\vec{\psi} ; \vec{\varphi} ; \Phi)=\mathbb{1}_{2}$. When we work in the bases $\vec{\gamma}$ and $\vec{\varphi}$ for the (co)homology groups, eq. (4.4) implies the following very compact formula for the coaction of every element of the associated period matrix,

$$
\Delta_{\epsilon} \int_{\gamma_{k}} \Phi \varphi_{l}=\int_{\gamma_{k}} \Phi \varphi_{1} \otimes \int_{\gamma_{1}} \Phi \varphi_{l}+\int_{\gamma_{k}} \Phi \varphi_{2} \otimes \int_{\gamma_{2}} \Phi \varphi_{l}
$$

We have checked that this coaction agrees with the condition (4.2) by computing the full period matrix through order $\epsilon^{4}$, and verifying that order by order in $\epsilon$ we reproduce the coaction $\Delta$ on MPLs.

We finish by writing an explicit formula for the coaction on the Gauss hypergeometric function ${ }_{2} F_{1}$, considering the parametric integral defined in eq. (5.1). Retaining the same bases of cycles $\vec{\gamma}$ and forms $\varphi$ in eqs. (5.4) and (5.11), respectively, where $C(\Omega(\vec{\gamma}) ; \vec{\varphi} ; \Phi)$ is the unit matrix, and using the general formula (4.1), the coaction takes the form

$$
\Delta_{\epsilon}\left(\int_{\gamma_{1}} \Phi \varphi\right)=\int_{\gamma_{1}} \Phi \varphi_{1} \otimes \int_{\gamma_{1}} \Phi \varphi+\int_{\gamma_{1}} \Phi \varphi_{2} \otimes \int_{\gamma_{2}} \Phi \varphi
$$

with $\Phi$ and $\varphi$ as in eq. (5.3). To reproduce the right-hand side of eq. (5.1), we then substitute $\alpha=1+n_{0}+a_{0} \epsilon, \beta=-n_{1 / x}-a_{1 / x} \epsilon, \gamma=2+n_{0}+n_{1}+\epsilon\left(a_{0}+a_{1}\right)$. Finally, using the coaction on Euler's beta function in eq. (4.6) and the relation $\Delta(f \cdot g)=\Delta(f) \cdot \Delta(g)$, we obtain the coaction on the Gauss hypergeometric function ${ }_{2} F_{1}$,

$$
\begin{aligned}
\Delta_{\epsilon}\left({ }_{2} F_{1}(\alpha, \beta ; \gamma ; x)\right)= & { }_{2} F_{1}(1+a \epsilon, b \epsilon ; 1+c \epsilon ; x) \otimes{ }_{2} F_{1}(\alpha, \beta ; \gamma ; x) \\
& -\frac{b \epsilon}{1+c \epsilon}{ }_{2} F_{1}(1+a \epsilon, 1+b \epsilon ; 2+c \epsilon ; x) \\
& \otimes \frac{\Gamma(1-\beta) \Gamma(\gamma)}{\Gamma(1-\beta+\alpha) \Gamma(\gamma-\alpha)} x^{1-\alpha}{ }_{2} F_{1}\left(\alpha, 1+\alpha-\gamma ; 1-\beta+\alpha ; \frac{1}{x}\right),
\end{aligned}
$$

where $\alpha=n_{\alpha}+a \epsilon, \beta=n_{\beta}+b \epsilon$ and $\gamma=n_{\gamma}+c \epsilon$ (in the notation of eq. (5.1), this means that $a=a_{0}, b=-a_{1 / x}$ and $\left.c=a_{0}+a_{1}\right)$.

\subsection{Coaction of a degenerate ${ }_{2} F_{1}$}

Our motivation for studying hypergeometric functions is that they appear when evaluating Feynman integrals in dimensional regularization. In these practical applications, one usually encounters non-generic hypergeometric functions, and it is thus important that one is able to handle these degenerate cases. There are two types of degenerations that can affect the general integrand of eq. (3.1). The first type occurs at special values of the external variables $x_{j}$, at which two or more of the polynomial factors coincide. The second type occurs at special values of the exponents $a_{I}$ in the twist $\Phi$, where any $a_{I}=0$ or where $\sum_{I} a_{I}=0$, which we excluded in section 3 because these cases require special treatment in 
the framework of twisted homology and cohomology. We expect that both types of degenerations can be handled by taking the corresponding limits of our general coaction formula. In this section we illustrate this in the context of ${ }_{2} F_{1}$, and derive coactions of degenerate cases by taking limits of eq. (5.14). We close this section by proposing that degeneracies of more general hypergeometric functions can be taken systematically through a detailed analysis of twisted cycles.

Special values of the variable. The first degeneration that we consider is when $x$ takes particular values. For ${ }_{2} F_{1}(\alpha, \beta ; \gamma ; x)$, the particular values are $x=0$, and $x=1$, when the factor $1-x u$ in the Euler integral representation eq. (5.1) combines with one of the others, reducing the number of branch points from four (including infinity) to three. We first consider $x \rightarrow 0$, in which case

$$
{ }_{2} F_{1}(\alpha, \beta ; \gamma ; 0)=1 .
$$

Taking the same limit on the right-hand side of eq. (5.14) we find that the first term gives $1 \otimes 1$ and the second term vanishes, reproducing the expected result

$$
\Delta_{\epsilon}\left({ }_{2} F_{1}(\alpha, \beta ; \gamma ; 0)\right)=1 \otimes 1
$$

A more interesting limit is when we set $x \rightarrow 1$, in which case

$$
{ }_{2} F_{1}(\alpha, \beta ; \gamma ; 1)=\frac{\Gamma(\gamma) \Gamma(\gamma-\alpha-\beta)}{\Gamma(\gamma-\alpha) \Gamma(\gamma-\beta)} .
$$

In this limit, the two terms in eq. (5.14) can be shown to reduce to

$$
\Delta_{\epsilon}\left(\frac{\Gamma(\gamma) \Gamma(\gamma-\alpha-\beta)}{\Gamma(\gamma-\alpha) \Gamma(\gamma-\beta)}\right)=\frac{\Gamma(1+c \epsilon) \Gamma(1+(c-a-b) \epsilon)}{\Gamma(1+(c-a) \epsilon) \Gamma(1+(c-b) \epsilon)} \otimes \frac{\Gamma(\gamma) \Gamma(\gamma-\alpha-\beta)}{\Gamma(\gamma-\alpha) \Gamma(\gamma-\beta)},
$$

where we recall that $\alpha=n_{\alpha}+a \epsilon$ and similarly for $\beta$ and $\gamma$. It is simple to verify that this coaction satisfies eq. (4.2) and is consistent with the coaction on the gamma function given in eq. (2.9).

Degenerate exponents. We consider the integrand in the Euler representation eq. (5.1) of ${ }_{2} F_{1}(\alpha, \beta ; \gamma ; x)$ to be degenerate if any of the three exponents is an integer, or if their sum is an integer. In terms of the function $\Phi$ given in eq. (5.3), these are the cases when any of $a_{0}, a_{1}, a_{1 / x}$ is 0 , or when $a_{0}+a_{1}+a_{1 / x}=0$. In these cases, $\Phi$ does not vanish nor is it singular at the corresponding point (or the point at infinity, in the case of the sum of the exponents being integer). Note also that it is precisely in these cases that the number of critical points of $\log \Phi$ is less than $2 .{ }^{6}$

Recall that in the notation of eq. (5.14),

$$
a_{0}=a, \quad a_{1}=c-a, \quad a_{1 / x}=-b, \quad a_{0}+a_{1}+a_{1 / x}=c-b .
$$

\footnotetext{
${ }^{6}$ However, it would be wrong to conclude that the dimension of (co)homology is less than 2, as the Morse theory arguments require nondegeneracy.
} 
So, in terms of $\alpha, \beta, \gamma$, the degenerations occur when any of $\alpha, \beta, \gamma-\alpha$ or $\gamma-\beta$ is an integer (this can also be seen directly from the integral representation in (2.12) or its symmetric version upon swapping $\alpha$ and $\beta$ ). While in principle we should check the coaction in each of these four cases as well as in the cases where more than one of the exponents is an integer, here we will simply discuss one such case as an example.

We consider the example of ${ }_{2} F_{1}(-\epsilon, 1 ; 1-\epsilon ; x)$, already introduced in eq. (2.13), for which we obtained a coaction by 'resumming' the Laurent expansion to all orders in $\epsilon$. In the notation of eq. (5.14) we have $a=-1, b=0, c=-1$. It is possible to apply eq. (5.14) with $b \neq 0$ and then take the limit $b \rightarrow 0$ to recover the correct coaction. We then obtain ${ }^{7}$

$$
\Delta_{\epsilon}\left({ }_{2} F_{1}(-\epsilon, 1 ; 1-\epsilon ; x)\right)=1 \otimes{ }_{2} F_{1}(-\epsilon, 1 ; 1-\epsilon ; x)-\frac{x \epsilon}{1-\epsilon}{ }_{2} F_{1}(1-\epsilon, 1 ; 2-\epsilon ; x) \otimes x^{\epsilon} .
$$

Noting that

$$
\frac{x \epsilon}{1-\epsilon}{ }_{2} F_{1}(1-\epsilon, 1 ; 2-\epsilon ; x)=F(\epsilon, x)=\sum_{n=1}^{\infty} \epsilon^{n} \operatorname{Li}_{n}(x),
$$

with $F(\epsilon, x)$ given in eq. (2.13), we find that the expression we obtained for a generic ${ }_{2} F_{1}$, eq. (5.14), reduces to eq. (2.14) in this degenerate limit.

While we have only discussed the consistency of our coaction with the degeneration of the exponents on an example, we have checked it on several other cases and believe it to be a general feature. Indeed, the degeneration of exponents can be dealt with through a careful analysis of twisted cycles [27]. In this paper, we have considered integrals of the form $\int_{\gamma} \Phi \varphi$, where $\Phi$ is the multi-valued function introducing the twist. Such integrals can equivalently be written as $\int_{\mathcal{C}} \varphi$, where $\mathcal{C}$ is a twisted cycle, a variant of $\gamma$ that contains a choice of branch for the function $\Phi$ and includes windings around the boundaries of $\gamma$, such that the boundary of $\mathcal{C}$ is zero. The windings are the key feature that makes it possible to take limits of integer exponents of $\Phi$. In such a limit, the twisted cycle reduces to a contour encircling the corresponding boundary or boundaries. The integral can then be evaluated simply by taking residues. We have confirmed that this procedure agrees with the degenerate coaction formulas we have found for ${ }_{2} F_{1}$.

\section{One-dimensional integrals: Lauricella functions $F_{D}^{(n)}$}

In this section we study the class of integrals called the Lauricella $F_{D}^{(n)}$ functions, represented by the one-dimensional integral:

$$
\int_{0}^{1} u^{\alpha-1}(1-u)^{\gamma-\alpha-1} \prod_{i=1}^{n}\left(1-x_{i} u\right)^{-\beta_{i}} d u=\frac{\Gamma(\alpha) \Gamma(\gamma-\alpha)}{\Gamma(\gamma)} F_{D}^{(n)}\left(\alpha ; \beta_{1}, \ldots, \beta_{n} ; \gamma ; x_{1}, \ldots, x_{n}\right) .
$$

The cases $n=0$ and $n=1$ correspond to $F_{D}^{(0)}(\alpha ; \gamma)=1$ and $F_{D}^{(1)}(\alpha ; \beta ; \gamma ; x)=$ ${ }_{2} F_{1}(\alpha, \beta ; \gamma ; x)$. Here, we first discuss the case $n=2$, which corresponds to the Appell $F_{1}$ function, and then consider the case of general $n$. The construction is a simple generalization of what we have seen in section 5 for ${ }_{2} F_{1}$. The twisted homology and cohomology groups associated with the Lauricella $F_{D}^{(n)}$ functions have been studied in ref. [60].

\footnotetext{
${ }^{7}$ In taking the limit $\{\beta \rightarrow 1, b \rightarrow 0\}$, we note that $b \epsilon \Gamma(1-\beta)=b \epsilon \Gamma(-b \epsilon) \rightarrow-1$. Furthermore, ${ }_{2} F_{1}(m, 0, n ; x)=1$ for any $m, n$.
} 


\subsection{The Appell $F_{1}$ function}

The parametric representation of the Appell $F_{1}$ function is obtained by setting $n=2$ in eq. (6.1):

$$
\int_{0}^{1} u^{\alpha-1}(1-u)^{\gamma-\alpha-1}(1-x u)^{-\beta}(1-y u)^{-\beta^{\prime}} d u=\frac{\Gamma(\alpha) \Gamma(\gamma-\alpha)}{\Gamma(\gamma)} F_{1}\left(\alpha ; \beta, \beta^{\prime} ; \gamma ; x, y\right),
$$

where we have set $x_{1}=x$ and $x_{2}=y$, and $\beta_{1}=\beta$ and $\beta_{2}=\beta^{\prime}$. To construct its coaction we will follow the same steps as for the ${ }_{2} F_{1}$ function and start from the parametric integral

$$
\int_{\gamma_{1}} \Phi \varphi=\int_{0}^{1} u^{n_{0}+a_{0} \epsilon}(1-u)^{n_{1}+a_{1} \epsilon}(1-x u)^{n_{1 / x}+a_{1 / x} \epsilon}(1-y u)^{n_{1 / y}+a_{1 / y} \epsilon} d u
$$

with $\gamma_{1}=[0,1]$ and $a_{i} \in \mathbb{C}^{*}$ and $n_{i} \in \mathbb{Z}$. In the notation of section 3 , we have

$$
\begin{aligned}
& \Phi=u^{a_{0} \epsilon}(1-u)^{a_{1} \epsilon}(1-x u)^{a_{1 / x} \epsilon}(1-y u)^{a_{1 / y} \epsilon} \\
& \varphi=u^{n_{0}}(1-u)^{n_{1}}(1-x u)^{n_{1 / x}}(1-y u)^{n_{1 / y}} d u .
\end{aligned}
$$

The underlying geometry is determined by the equations

$$
P_{0}(u)=u, \quad P_{1}(u)=1-u, \quad P_{1 / x}(u)=1-x u, \quad P_{1 / y}(u)=1-y u .
$$

The hyperplanes are points on the real line:

$$
H_{0}=\{u=0\}, \quad H_{1}=\{u=1\}, \quad H_{1 / x}=\{u=1 / x\}, \quad H_{1 / y}=\{u=1 / y\} .
$$

The index of the $P_{i}$ and the $H_{i}$ carries the information of which factor in $\Phi$ it is associated with. The dimension of the (co)homology groups can be determined in different ways: we can either count the solutions to $d \log \Phi=0$, or, alternatively, count the bounded chambers defined by the hyperplanes above. One may also consider the basis of integer-shift relations, which can be derived using integration-by-parts identities. In any case we find that the dimension is three. As a basis of of the homology group, we can choose the cycles

$$
\gamma_{1}=[0,1], \quad \gamma_{1 / x}=[0,1 / x], \quad \gamma_{1 / y}=[0,1 / y] .
$$

The index of the cycles carries the information of which hyperplanes make up its boundary.

The cycles $\gamma_{i}$ are positive geometries with canonical forms $\Omega\left(\gamma_{i}\right)$ (see eq. (3.11)). The forms $\Omega\left(\gamma_{1}\right), \Omega\left(\gamma_{1 / x}\right)$ and $\Omega\left(\gamma_{1 / y}\right)$ are a basis of the cohomology group. However, building on the experience of the ${ }_{2} F_{1}$ function, we prefer to choose an orthonormal basis that generalizes that of section 5.2. For the present example, we thus choose the forms

$$
\varphi_{1}=a_{1} \epsilon \frac{d u}{1-u}, \quad \varphi_{1 / x}=a_{1 / x} x \epsilon \frac{d u}{1-u x}, \quad \varphi_{1 / y}=a_{1 / y} y \epsilon \frac{d u}{1-u y} .
$$

With this basis of $d \log$-forms and the cycles $\vec{\gamma}$ of eq. (6.7), we find that the matrix of intersection numbers $C(\Omega(\vec{\gamma}) ; \vec{\varphi} ; \Phi)$ is the identity matrix $\mathbb{1}_{3}$.

Using eq. (4.1), we can then write the coaction for the parametric integral in eq. (6.3):

$$
\Delta_{\epsilon} \int_{\gamma_{1}} \Phi \varphi=\int_{\gamma_{1}} \Phi \varphi_{1} \otimes \int_{\gamma_{1}} \Phi \varphi+\int_{\gamma_{1}} \Phi \varphi_{1 / x} \otimes \int_{\gamma_{1 / x}} \Phi \varphi+\int_{\gamma_{1}} \Phi \varphi_{1 / y} \otimes \int_{\gamma_{1 / y}} \Phi \varphi .
$$


Finally, to write the coaction on the Appell $F_{1}$ functions, we simply need to rewrite the above integrals in terms of the $F_{1}$ functions and use the coaction on the beta function we have established in eq. (4.6). Explicitly, we have

$$
\Phi \varphi=u^{\alpha-1}(1-u)^{\gamma-\alpha-1}(1-x u)^{-\beta}(1-y u)^{-\beta^{\prime}} d u,
$$

with

$$
\begin{aligned}
\int_{\gamma_{1}} \Phi \varphi & =\frac{\Gamma(\alpha) \Gamma(\gamma-\alpha)}{\Gamma(\gamma)} F_{1}\left(\alpha ; \beta, \beta^{\prime} ; \gamma ; x, y\right), \\
\int_{\gamma_{1 / x}} \Phi \varphi & =x^{-\alpha} \frac{\Gamma(\alpha) \Gamma(1-\beta)}{\Gamma(1+\alpha-\beta)} F_{1}\left(\alpha ; 1+\alpha-\gamma, \beta^{\prime} ; 1+\alpha-\beta ; \frac{1}{x}, \frac{y}{x}\right), \\
\int_{\gamma_{1 / y}} \Phi \varphi & =y^{-\alpha} \frac{\Gamma(\alpha) \Gamma\left(1-\beta^{\prime}\right)}{\Gamma\left(1+\alpha-\beta^{\prime}\right)} F_{1}\left(\alpha ; \beta, 1+\alpha-\gamma ; 1+\alpha-\beta^{\prime} ; \frac{x}{y}, \frac{1}{y}\right),
\end{aligned}
$$

with the identification $\alpha=1+n_{0}+a_{0} \epsilon, \gamma=2+n_{0}+n_{1}+\left(a_{0}+a_{1}\right) \epsilon, \beta=-n_{1 / x}-a_{1 / x} \epsilon$ and $\beta^{\prime}=-n_{1 / y}-a_{1 / y} \epsilon$. The integrals with the forms $\varphi_{i}$ in the integrand appearing in the left entries of the coaction (6.9) are easily obtained as special cases of these expressions.

To check that the coaction in eq. (6.9) is consistent with eq. (4.2), we have computed the full period matrix $P(\vec{\gamma} ; \vec{\varphi} ; \Phi)$ with $\vec{\gamma}$ in (6.7) and $\vec{\varphi}$ in (6.8), and its Laurent expansion through order $\epsilon^{4}$, i.e., through weight 4 (given that we have chosen a basis of $d \log$-forms, the power of $\epsilon$ and the transcendental weight are aligned). The computations were done using the Mathematica package PolyLogTools [59]. We then checked that the coaction on the period matrix in eq. (4.4) satisfies eq. (4.2) at each order in $\epsilon$. Furthermore, it is easy to check that the coaction we have constructed for the Appell $F_{1}$ functions degenerates to the appropriate coactions (that of the Gauss hypergeometric function or that of the beta function) when $x$ or $y$ are set to 0 or 1 .

\subsection{Coaction for generic Lauricella $F_{D}^{(n)}$}

It is straightforward to generalize the analysis of the Appell $F_{1}$ function from its representation as a one-dimensional integral to the generic Lauricella function $F_{D}^{(n)}$ as defined in eq. (6.1). The multivalued part of the integrand is given by

$$
\Phi=u^{a \epsilon} \prod_{i=0}^{n}\left(1-x_{i} u\right)^{c_{i} \epsilon},
$$

where we define $x_{0}=1$, and $a, c_{i} \in \mathbb{C}^{*}$. The single-valued 1 -form is

$$
\varphi=u^{m} \prod_{i=0}^{n}\left(1-x_{i} u\right)^{n_{i}} d u,
$$

with $m, n_{i} \in \mathbb{Z}$. It is easy to see that there are $n+1$ solutions to $d \log \Phi=0$ and the dimension of the (co)homology groups is thus $n+1$.

A basis of the homology group is formed by the cycles $\gamma_{i} \in \vec{\gamma}$ with

$$
\gamma_{i}=\left[0,1 / x_{i}\right], \quad i=0, \ldots, n .
$$


As in the previous example, we choose a basis $\vec{\varphi}$ of the cohomology group such that $C(\Omega(\vec{\gamma}) ; \vec{\varphi} ; \Phi)$ is the unit matrix $\mathbb{1}_{n+1}$. Such a basis is given by $\varphi_{i} \in \vec{\varphi}$ with

$$
\varphi_{i}=c_{i} x_{i} \epsilon \frac{d u}{1-x_{i} u} .
$$

It then follows from eq. (4.1) that

$$
\Delta_{\epsilon} \int_{\gamma_{0}} \Phi \varphi=\sum_{i=0}^{n} \int_{\gamma_{0}} \Phi \varphi_{i} \otimes \int_{\gamma_{i}} \Phi \varphi .
$$

To obtain the coaction on the Lauricella functions in (6.1) from this expression, we use

$$
\Phi \varphi=u^{\alpha-1}(1-u)^{\gamma-\alpha-1} \prod_{i=1}^{n}\left(1-x_{i} u\right)^{-\beta_{i}} d u,
$$

with

$$
\begin{aligned}
\int_{\gamma_{0}} \Phi \varphi= & \frac{\Gamma(\alpha) \Gamma(\gamma-\alpha)}{\Gamma(\gamma)} F_{D}^{(n)}\left(\alpha ; \beta_{1}, \ldots, \beta_{n} ; \gamma ; x_{1}, \ldots, x_{n}\right), \\
\int_{\gamma_{i}} \Phi \varphi= & x_{i}^{-\alpha} \frac{\Gamma(\alpha) \Gamma\left(1-\beta_{i}\right)}{\Gamma\left(1+\alpha-\beta_{i}\right)} \\
& F_{D}^{(n)}\left(\alpha ; \beta_{1}, \ldots, \beta_{i-1}, 1+\alpha-\gamma, \beta_{i+1}, \ldots \beta_{n} ; 1+\alpha-\beta_{i} ; y_{1}^{(i)}, \ldots, y_{n}^{(i)}\right), \quad \text { for } i \geq 1,
\end{aligned}
$$

where $y_{j}^{(i)}=x_{j} / x_{i}$ if $j \neq i$ and $y_{i}^{(i)}=1 / x_{i}$, together with the identifications $\alpha=1+m+a \epsilon$, $\gamma=2+m+n_{0}+\left(a+c_{0}\right) \epsilon, \beta_{i}=-n_{i}-c_{i} \epsilon$. It is straightforward to check the consistency of this result with the coactions obtained previously for the beta function, the Gauss hypergeometric function and the Appell $F_{1}$ function.

\section{Two-dimensional integrals: Appell functions}

In this section we study a family of functions, the so-called Appell functions $F_{1}, F_{2}, F_{3}$ and $F_{4}$. We have already encountered the $F_{1}$ function in the previous section, as it also admits a one-dimensional integral representation. We now study it from a different perspective. All Appell functions depend on two external variables (which we always denote $x$ and $y$ ) and admit a two-dimensional integral representation, see e.g. ref. [61]. The Appell $F_{1}$ is a special case of the Lauricella $F_{D}^{(n)}$ function with $n=2$, and it is known that these functions have both one-dimensional and $n$-dimensional integral representations [62]. This function therefore provides a natural and simple setting to illustrate the generalization of our discussion of previous sections beyond one dimension.

In principle this generalization is straightforward, as our framework is in no way restricted to one-dimensional integrals. The underlying geometry is now more involved, because it is no longer defined by points on a line but rather by lines in $\mathbb{P}^{2}(\mathbb{R})$. We can, nevertheless, proceed in the same way as for one-dimensional integrals: we construct bases of the associated homology and cohomology groups, and the coaction is immediately given 
by eq. (4.1). However, there is an interesting aspect related to the choice of bases that we wish to highlight: in the examples of the Gauss hypergeometric function and the Lauricella $F_{D}^{(n)}$ function, it was always straightforward to express all entries in the coaction in terms of the same class of function. As we will see in subsequent sections, this remains true for all Appell functions. Indeed - while not always straightforward to achieve in practice - with a properly chosen basis we will be able to express all entries of the period matrix of each Appell function in terms of the same type of function. That this should be possible in the first place can be understood to be a consequence of the fact that each of the Appell functions can be defined by a particular system of linear differential equations (see section 7.3). A proof of this statement in twisted de Rham theory was given in [63] for the Lauricella functions $F_{A}, F_{B}, F_{C}, F_{D}$, which include the Appell functions as special cases, and for ${ }_{p+1} F_{p}$. A suitable choice of bases of forms and cycles will be discussed in each example in turn.

We begin by considering the Appell $F_{1}$ function written as a two-dimensional integral, which we can explicitly compare with the coaction derived in section 6.1 where we used a one-dimensional representation. We proceed in section 7.2 with the Appell $F_{3}$ function, which has a more complex geometry. Next, we consider the Appell $F_{2}$ function in section 7.3, where expressing the right entries in the coaction in terms of Appell $F_{2}$ functions requires a somewhat intricate choice of basis; we use the basis of cycles introduced in ref. [64]. We conclude this section by considering the Appell $F_{4}$ function in section 7.4, where we will use a basis of cycles introduced in ref. [65].

\subsection{The Appell $F_{1}$ function as a double integral}

The Appell $F_{1}$ function can be written as a two-dimensional integral as follows:

$$
\begin{aligned}
& \int_{0}^{1} d v \int_{0}^{1-v} d u u^{\beta-1} v^{\beta^{\prime}-1}(1-u-v)^{\gamma-\beta-\beta^{\prime}-1}(1-x u-y v)^{-\alpha}= \\
& \quad=\frac{\Gamma(\beta) \Gamma\left(\beta^{\prime}\right) \Gamma\left(\gamma-\beta-\beta^{\prime}\right)}{\Gamma(\gamma)} F_{1}\left(\alpha, \beta, \beta^{\prime}, \gamma ; x, y\right) .
\end{aligned}
$$

In the notation of section 3.1, the multivalued part of the integrand is given by

$$
\Phi=u^{c_{1} \epsilon} v^{c_{2} \epsilon}(1-u-v)^{c_{3} \epsilon}(1-x u-y v)^{c_{4} \epsilon},
$$

and the single-valued 2 -form is

$$
\varphi=u^{n_{1}} v^{n_{2}}(1-u-v)^{n_{3}}(1-x u-y v)^{n_{4}} d u \wedge d v .
$$

The underlying geometry of the parametric integral in eq. (7.1) is determined by the hyperplanes

$$
H_{1}=\{u=0\}, H_{2}=\{v=0\}, H_{3}=\{1-u-v=0\}, H_{4}=\{1-x u-y v=0\},
$$

which we have represented in figure 1. It is clear from this picture that there are three independent bounded chambers, which means that the dimension of the second (co)homology 


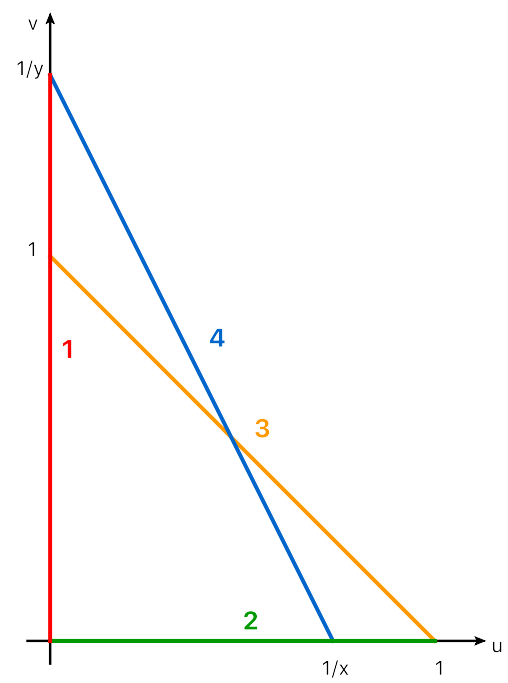

Figure 1. Geometry for the two-dimensional integral representation of the Appell $F_{1}$ integral of eq. (7.1).

groups is 3, as already established in section 6.1. We denote by $\gamma_{a b c}$ the cycle that is bounded by $H_{a}, H_{b}$, and $H_{c}$. As a basis we take the following three triangular regions:

$$
\begin{aligned}
\gamma_{123} & =\{0<u<1-v, \quad 0<v<1\}, \\
\gamma_{124} & =\{0<u<(1-y v) / x, \quad 0<v<1 / y\}, \\
\gamma_{234} & =\{(1-y v) / x<u<1-v, \quad 0<v<(x-1) /(x-y)\},
\end{aligned}
$$

where the inequalities on the right-hand sides are written under the assumption that, as in figure $1, x>1>y>0 .{ }^{8}$ The cycle $\gamma_{123}$ is the integration contour that appears in the representation of the $F_{1}$ function as a double integral, see eq. (7.1).

The cycles $\gamma_{a b c}$ define positive geometries with associated canonical forms. For instance,

$$
\Omega\left(\gamma_{123}\right)=d \log \frac{u}{1-u-v} \wedge d \log \frac{1-u-v}{v}=-\frac{d u \wedge d v}{u v(1-u-v)} .
$$

As in the case of one-dimensional integrals, choosing $\Omega\left(\gamma_{123}\right), \Omega\left(\gamma_{124}\right)$ and $\Omega\left(\gamma_{234}\right)$ as a basis of the cohomology would lead to a matrix $C(\Omega(\vec{\gamma}) ; \Omega(\vec{\gamma}) ; \Phi)$ with off-diagonal elements. We thus prefer to work with the alternative basis

$$
\begin{aligned}
& \varphi_{13}=c_{1} c_{3} \epsilon^{2} d \log (1-u-v) \wedge d \log (u)=\frac{c_{1} c_{3} \epsilon^{2} d u \wedge d v}{u(1-u-v)}, \\
& \varphi_{14}=c_{1} c_{4} \epsilon^{2} d \log (1-x u-y v) \wedge d \log (u)=\frac{c_{1} c_{4} \epsilon^{2} y d u \wedge d v}{u(1-x u-y v)}, \\
& \varphi_{34}=c_{3} c_{4} \epsilon^{2} d \log (1-u-v) \wedge d \log (1-x u-y v)=\frac{c_{3} c_{4} \epsilon^{2}(y-x) d u \wedge d v}{(1-u-v)(1-x u-y v)},
\end{aligned}
$$

\footnotetext{
${ }^{8}$ We chose this configuration so that the intersection of the hyperplanes $H_{3}$ and $H_{4}$ is inside the first quadrant of the $(u, v)$ plane. This leads to a pole associated with the factor $1-x u-y v$ inside the standard integration region, $\gamma_{123}$. Whenever such an issue arises, we assume that $x$ and $y$ have small positive imaginary parts so that the pole is shifted into the complex plane and the integral is well defined.
} 
where once again we use the indices of the hyperplanes $H_{a}$ to label the differential forms, i.e., the differential form $\varphi_{a b}$ has logarithmic singularities on the hyperplanes $H_{a}$ and $H_{b}$. The normalization of the $d \log$-forms is such that the intersection matrix $C(\Omega(\vec{\gamma}) ; \vec{\varphi} ; \Phi)$ is the identity matrix $\mathbb{1}_{3}$. These normalizations can be easily determined from eq. (3.24) and the observation that the leading poles of the period matrix correspond to the points where both integration variables have an endpoint singularity. To be more concrete, consider the contour $\gamma_{123}$, which gives rise to potential double endpoint singularities at $(u, v)=(0,0)$, $(0,1)$ and $(1,0)$ (corresponding to the intersections of $H_{1}$ and $H_{2}, H_{1}$ and $H_{3}$, and $H_{2}$ and $H_{3}$ respectively, see figure 1$)$. It is then easy to see that there is only a double endpoint singularity for the form $\varphi_{13}$ at $(u, v)=(0,1)$. The singularity is regulated by the factors $u^{c_{1} \epsilon}$ and $(1-u-v)^{c_{3} \epsilon}$ in $\Phi$. The normalization factor $c_{1} c_{3} \epsilon^{2}$ for $\varphi_{13}$ in eq. (7.7) then guarantees that

$$
\int_{\gamma_{123}} \Phi \varphi_{13}=1+\mathcal{O}(\epsilon)
$$

For the forms $\varphi_{14}$ and $\varphi_{34}$ no double endpoint singularity is generated, and hence, with the normalization factors in eq. (7.7), we have:

$$
\int_{\gamma_{123}} \Phi \varphi_{14}=0+\mathcal{O}(\epsilon) \text { and } \int_{\gamma_{123}} \Phi \varphi_{34}=0+\mathcal{O}(\epsilon) .
$$

A similar situation holds for $\gamma_{124}$ and $\gamma_{234}$ : for the former a double pole is generated only for the form $\varphi_{14}$ in eq. (7.7), while for the latter only for the form $\varphi_{34}$. Alternatively, we can use eq. (3.23) to determine the matrix of intersection numbers (note, however, that this formula requires the evaluation of the critical points, which are often nontrivial functions of the $c_{i}$ ).

The coaction on the $F_{1}$ function can then be obtained using

$$
\Delta_{\epsilon} \int_{\gamma_{123}} \Phi \varphi=\int_{\gamma_{123}} \Phi \varphi_{13} \otimes \int_{\gamma_{123}} \Phi \varphi+\int_{\gamma_{123}} \Phi \varphi_{14} \otimes \int_{\gamma_{124}} \Phi \varphi+\int_{\gamma_{123}} \Phi \varphi_{34} \otimes \int_{\gamma_{234}} \Phi \varphi,
$$

with

$$
\Phi \varphi=u^{\beta-1} v^{\beta^{\prime}-1}(1-u-v)^{\gamma-\beta-\beta^{\prime}-1}(1-x u-y v)^{-\alpha}
$$

and

$$
\begin{aligned}
\int_{\gamma_{123}} \Phi \varphi= & \frac{\Gamma(\beta) \Gamma\left(\beta^{\prime}\right) \Gamma\left(\gamma-\beta-\beta^{\prime}\right)}{\Gamma(\gamma)} F_{1}\left(\alpha, \beta, \beta^{\prime}, \gamma ; x, y\right) \\
\int_{\gamma_{124}} \Phi \varphi= & x^{-\beta} y^{-\beta^{\prime}} \frac{\Gamma(\beta) \Gamma\left(\beta^{\prime}\right) \Gamma(1-\alpha)}{\Gamma\left(1-\alpha+\beta+\beta^{\prime}\right)} F_{1}\left(1+\beta+\beta^{\prime}-\gamma, \beta, \beta^{\prime}, 1-\alpha+\beta+\beta^{\prime} ; \frac{1}{x}, \frac{1}{y}\right), \\
\int_{\gamma_{234}} \Phi \varphi= & x^{1+\beta^{\prime}-\gamma}(x-1)^{\gamma-\alpha-\beta}(x-y)^{-\beta^{\prime}} e^{i \pi \alpha} \frac{\Gamma(1-\alpha) \Gamma\left(\beta^{\prime}\right) \Gamma\left(\gamma-\beta-\beta^{\prime}\right)}{\Gamma(1-\alpha-\beta+\gamma)} \\
& F_{1}\left(1-\beta, 1-\alpha, \beta^{\prime}, 1-\alpha-\beta+\gamma ; 1-x, \frac{y(x-1)}{x-y}\right)
\end{aligned}
$$

with the identifications $n_{1}+c_{1} \epsilon=\beta-1, n_{2}+c_{2} \epsilon=\beta^{\prime}-1, n_{3}+c_{3} \epsilon=\gamma-\beta-\beta^{\prime}-1$ and $n_{4}+c_{4} \epsilon=-\alpha$. We note that for this example it was straightforward to write all integrals 
in eq. (7.12) as members of the $F_{1}$ family through simple changes of variables. We have explicitly computed the period matrix $P(\vec{\gamma} ; \vec{\varphi} ; \Phi)$ and checked that the relation in eq. (4.2) is satisfied order by order in $\epsilon$ through weight 4 .

The coaction for the Appell $F_{1}$ function constructed from the two-dimensional integral representation in eq. (7.10) is equivalent to the one we constructed in section 6.1 from the one-dimensional representation. To be more precise, the period matrix of section 6.1, which we denote $P_{6.1}$, is related to the one computed in this section, which we denote $P_{7.1}$, as follows:

$$
P_{6.1}=M \cdot P_{7.1} \cdot M^{-1}
$$

with

$$
M=\left(\begin{array}{ccc}
1 & 0 & 0 \\
1 & 0 & -\frac{a}{b} \\
\frac{b^{\prime}-a}{b^{\prime}} & \frac{a}{b^{\prime}} & \frac{a}{b^{\prime}}
\end{array}\right)
$$

where $a, b$ and $b^{\prime}$ are the coefficients of $\epsilon$ in $\alpha, \beta$ and $\beta^{\prime}$ respectively, e.g., $\alpha=n_{\alpha}+a \epsilon$ with $n_{\alpha} \in \mathbb{Z}$. The matrix $M$ can be interpreted as encoding the relation between the bases of integrands used in section 6.1 and in the present section. For a given contour, the relations we obtain from eq. (7.13) are special cases of the well-known integer-shift relations (see e.g. ref. [66]).

\subsection{The Appell $F_{3}$ function}

The next example we consider is the Appell $F_{3}$ function, which has the following twodimensional integral representation:

$$
\begin{aligned}
& \int_{0}^{1} d v \int_{0}^{1-v} d u u^{\beta-1} v^{\beta^{\prime}-1}(1-u-v)^{\gamma-\beta-\beta^{\prime}-1}(1-x u)^{-\alpha}(1-y v)^{-\alpha^{\prime}}= \\
& \quad=\frac{\Gamma(\beta) \Gamma\left(\beta^{\prime}\right) \Gamma\left(\gamma-\beta-\beta^{\prime}\right)}{\Gamma(\gamma)} F_{3}\left(\alpha, \alpha^{\prime}, \beta, \beta^{\prime}, \gamma ; x, y\right) .
\end{aligned}
$$

This example does not introduce any conceptually new features, but has a slightly more complicated geometry when compared to the case of the $F_{1}$ function, as it has five factors in the integrand.

As usual, we define

$$
\begin{aligned}
& \Phi=u^{c_{1} \epsilon} v^{c_{2} \epsilon}(1-x u)^{c_{3} \epsilon}(1-y v)^{c_{4} \epsilon}(1-u-v)^{c_{5} \epsilon}, \\
& \varphi=u^{n_{1}} v^{n_{2}}(1-x u)^{n_{3}}(1-y v)^{n_{4}}(1-u-v)^{n_{5}} d u \wedge d v .
\end{aligned}
$$

The geometry underlying the Appell $F_{3}$ function is then determined by the hyperplanes

$$
\begin{array}{ll}
H_{1}=\{u=0\}, & H_{2}=\{v=0\}, \quad H_{3}=\{1-x u=0\}, \\
H_{4}=\{1-y v=0\}, & H_{5}=\{1-u-v=0\},
\end{array}
$$

which we represent in figure 2 for $x>y>1$ (a similar comment as the one in footnote 8 applies). The dimension of the (co)homology groups can be determined by counting the critical points of $\Phi$ or by counting the independent bounded chambers in figure 2. Either way, we find the dimension to be 4 . 


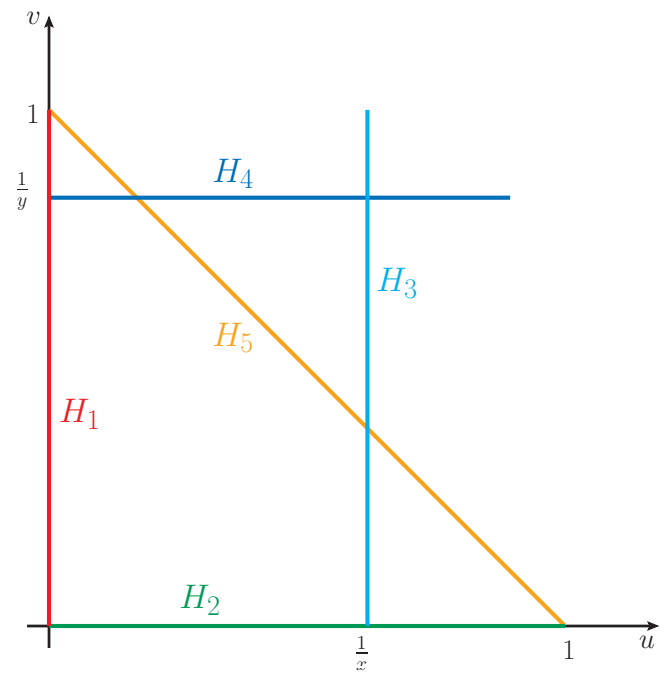

Figure 2. Geometry for the Appell $F_{3}$ integral representation in eq. (7.15).

Similarly to the Appell $F_{1}$ function case, we choose triangular cycles $\gamma_{a b c}$ defined by the hyperplanes $H_{a}, H_{b}$ and $H_{c}$ as a basis of the homology group. In the present case, we choose $\gamma_{125}, \gamma_{235}, \gamma_{345}$ and $\gamma_{145}$. The contour $\gamma_{125}$ is the standard 2-simplex in the definition of the $F_{3}$ function given in eq. (7.15). These four cycles are all positive geometries and their canonical forms provide a basis of the cohomology group. As in previous examples, we use a different basis, in this case

$$
\begin{aligned}
& \varphi_{12}=c_{1} c_{2} \epsilon^{2} d \log u \wedge d \log v=\frac{c_{1} c_{2} \epsilon^{2} d u \wedge d v}{u v}, \\
& \varphi_{23}=c_{2} c_{3} \epsilon^{2} d \log (1-x u) \wedge d \log v=-\frac{c_{2} c_{3} \epsilon^{2} x d u \wedge d v}{(1-x u) v}, \\
& \varphi_{34}=c_{3} c_{4} \epsilon^{2} d \log (1-x u) \wedge d \log (1-y v)=\frac{c_{3} c_{4} \epsilon^{2} x y d u \wedge d v}{(1-x u)(1-y v)}, \\
& \varphi_{14}=c_{1} c_{4} \epsilon^{2} d \log (u) \wedge d \log (1-y v)=-\frac{c_{1} c_{4} \epsilon^{2} y d u \wedge d v}{u(1-y v)},
\end{aligned}
$$

which gives $C(\Omega(\vec{\gamma}) ; \vec{\varphi} ; \Phi)=\mathbb{1}_{4}$. The normalization of the $d$ log-forms is determined as described in the $F_{1}$ example in the discussion below eq. (7.7) above.

It is straightforward to write all the entries of the period matrix $P(\vec{\gamma} ; \vec{\varphi} ; \Phi)$ entirely in terms of $F_{3}$ functions, since we can find changes of variables mapping the arrangement of hyperplanes into itself while also mapping $\gamma_{125}$ into any of the other basis cycles. We thus obtain:

$$
\begin{aligned}
\int_{\gamma_{125}} \Phi \varphi= & \frac{\Gamma(\beta) \Gamma\left(\beta^{\prime}\right) \Gamma\left(\gamma-\beta-\beta^{\prime}\right)}{\Gamma(\gamma)} F_{3}\left(\alpha, \alpha^{\prime}, \beta, \beta^{\prime}, \gamma ; x, y\right) \\
\int_{\gamma_{235}} \Phi \varphi= & (x-1)^{\gamma-\beta-\alpha} x^{1-\gamma} e^{i \pi \alpha} \frac{\Gamma(1-\alpha) \Gamma\left(\beta^{\prime}\right) \Gamma\left(\gamma-\beta-\beta^{\prime}\right)}{\Gamma(1-\alpha-\beta+\gamma)} \\
& \times F_{3}\left(1-\beta, \alpha^{\prime}, 1-\alpha, \beta^{\prime}, 1-\alpha-\beta+\gamma ; 1-x, \frac{x-1}{x} y\right)
\end{aligned}
$$




$$
\begin{aligned}
\int_{\gamma_{145}} \Phi \varphi= & (y-1)^{\gamma-\beta^{\prime}-\alpha^{\prime}} y^{1-\gamma} e^{i \pi \alpha^{\prime}} \frac{\Gamma(\beta) \Gamma\left(1-\alpha^{\prime}\right) \Gamma\left(\gamma-\beta-\beta^{\prime}\right)}{\Gamma\left(1-\alpha^{\prime}-\beta^{\prime}+\gamma\right)} \\
& \times F_{3}\left(\alpha, 1-\beta^{\prime}, \beta, 1-\alpha^{\prime}, 1-\alpha^{\prime}-\beta^{\prime}+\gamma ; \frac{y-1}{y} x, 1-y\right) \\
\int_{\gamma_{345}} \Phi \varphi= & -\frac{x^{\alpha^{\prime}+\beta^{\prime}-\gamma} y^{\alpha+\beta-\gamma^{\prime}} e^{i \pi\left(\gamma-\beta-\beta^{\prime}\right)}}{(x+y-x y)^{\alpha+\alpha^{\prime}+\beta+\beta^{\prime}-\gamma-1}} \frac{\Gamma(1-\alpha) \Gamma\left(1-\alpha^{\prime}\right) \Gamma\left(\gamma-\beta-\beta^{\prime}\right)}{\Gamma\left(2+\gamma-\alpha-\alpha^{\prime}-\beta-\beta^{\prime}\right)} \\
& \times F_{3}\left(1-\beta, 1-\beta^{\prime}, 1-\alpha, 1-\alpha^{\prime}, 2+\gamma-\alpha-\alpha^{\prime}-\beta-\beta^{\prime} ; \frac{x+y-x y}{y}, \frac{x+y-x y}{x}\right)
\end{aligned}
$$

with the identifications $n_{1}+c_{1} \epsilon=\beta-1, n_{2}+c_{2} \epsilon=\beta^{\prime}-1, n_{3}+c_{3} \epsilon=-\alpha, n_{4}+c_{4} \epsilon=-\alpha^{\prime}$, and $n_{5}+c_{5} \epsilon=\gamma-\beta-\beta^{\prime}-1$.

The coaction on the $F_{3}$ function then follows from eq. (4.1), which yields

$$
\begin{aligned}
\Delta_{\epsilon} \int_{\gamma_{125}} \Phi \varphi= & \int_{\gamma_{125}} \Phi \varphi_{12} \otimes \int_{\gamma_{125}} \Phi \varphi+\int_{\gamma_{125}} \Phi \varphi_{23} \otimes \int_{\gamma_{235}} \Phi \varphi \\
& +\int_{\gamma_{125}} \Phi \varphi_{34} \otimes \int_{\gamma_{345}} \Phi \varphi+\int_{\gamma_{125}} \Phi \varphi_{14} \otimes \int_{\gamma_{145}} \Phi \varphi .
\end{aligned}
$$

We have explicitly checked that this coaction satisfies the relation in eq. (4.2) for all entries of the period matrix $P(\vec{\gamma} ; \vec{\varphi} ; \Phi)$ through weight 4 .

The Appell $F_{3}$ function is the special case with $n=2$ of the Lauricella series $F_{B}^{(n)}$, and this construction of the coaction generalizes straightforwardly to all $n$.

\subsection{The Appell $F_{2}$ function}

The Appell $F_{2}$ function can be written as a two-dimensional integral as follows:

$$
\begin{aligned}
& \int_{0}^{1} d v \int_{0}^{1} d u u^{\beta-1} v^{\beta^{\prime}-1}(1-u)^{\gamma-\beta-1}(1-v)^{\gamma^{\prime}-\beta^{\prime}-1}(1-x u-v y)^{-\alpha}= \\
& \quad=\frac{\Gamma(\beta) \Gamma\left(\beta^{\prime}\right) \Gamma(\gamma-\beta) \Gamma\left(\gamma^{\prime}-\beta^{\prime}\right)}{\Gamma(\gamma) \Gamma\left(\gamma^{\prime}\right)} F_{2}\left(\alpha ; \beta, \beta^{\prime} ; \gamma, \gamma^{\prime} ; x, y\right) .
\end{aligned}
$$

We follow the same steps as we did for the Appell $F_{3}$ function, and define

$$
\begin{aligned}
& \Phi=u^{c_{1} \epsilon} v^{c_{2} \epsilon}(1-u)^{c_{3} \epsilon}(1-v)^{c_{4} \epsilon}(1-u x-v y)^{c_{5} \epsilon}, \\
& \varphi=u^{n_{1}} v^{n_{2}}(1-u)^{n_{3}}(1-v)^{n_{4}}(1-u x-v y)^{n_{5}} d u \wedge d v .
\end{aligned}
$$

The underlying geometry is the same as that of the Appell $F_{3}$ case studied in the previous section, as can be seen in figure 3 where we have chosen values of $x$ and $y$ that make the equivalence clear. The geometry is determined by the hyperplanes

$$
\begin{array}{ll}
H_{1}=\{u=0\}, & H_{2}=\{v=0\}, \quad H_{3}=\{1-u=0\}, \\
H_{4}=\{1-v=0\}, & H_{5}=\{1-u x-v y=0\},
\end{array}
$$

which we represent in figure 3 for $0<x, y<1$. It is clear that there are four bounded chambers, which implies that the dimension of the (co)homology groups is 4 . We choose 


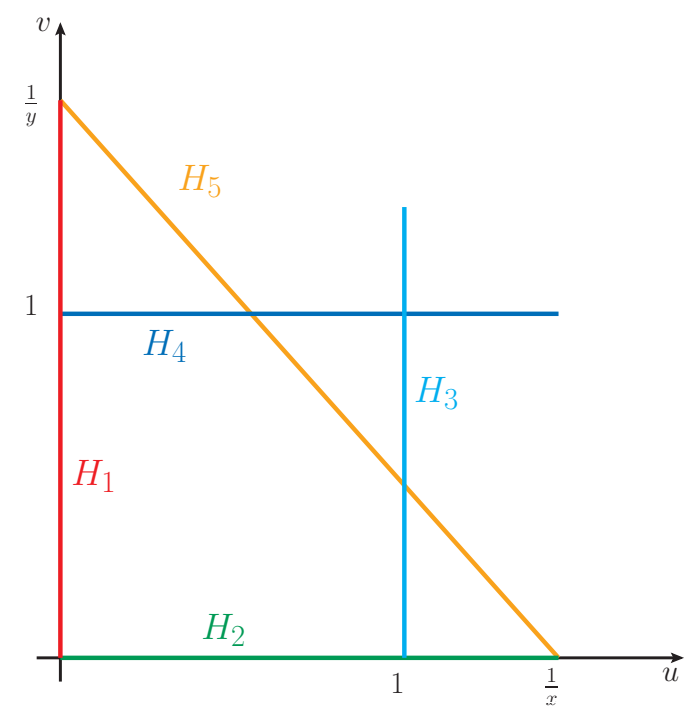

Figure 3. Geometry for the Appell $F_{2}$ integral representation of eq. (7.21).

the cycles $\gamma_{125}, \gamma_{235}, \gamma_{345}$ and $\gamma_{145}$ as generators of the homology group, where we recall that the indices correspond to the hypersurfaces $H_{i}$ that delimit each cycle. This choice does not include the unit square $\gamma_{1234}$ that appears in the definition of the $F_{2}$ in eq. (7.21), but it can be easily obtained from

$$
\gamma_{1234}=\gamma_{125}+\gamma_{345}-\gamma_{145}-\gamma_{235} .
$$

As a basis of the cohomology group, we choose the forms

$$
\begin{aligned}
& \varphi_{12}=c_{1} c_{2} \epsilon^{2} d \log (u) \wedge d \log (v)=\frac{c_{1} c_{2} \epsilon^{2} d u \wedge d v}{u v}, \\
& \varphi_{23}=c_{2} c_{3} \epsilon^{2} d \log (1-u) \wedge d \log (v)=-\frac{c_{2} c_{3} \epsilon^{2} d u \wedge d v}{(1-u) v}, \\
& \varphi_{34}=c_{3} c_{4} \epsilon^{2} d \log (1-u) \wedge d \log (1-v)=\frac{c_{3} c_{4} \epsilon^{2} d u \wedge d v}{(1-u)(1-v)} \\
& \varphi_{14}=c_{1} c_{4} \epsilon^{2} d \log (u) \wedge d \log (1-v)=-\frac{c_{1} c_{4} \epsilon^{2} d u \wedge d v}{u(1-v)}
\end{aligned}
$$

which gives $C(\Omega(\vec{\gamma}) ; \vec{\varphi} ; \Phi)=\mathbb{1}_{4}$.

With these definitions, the coaction on the Appell $F_{2}$ function can be written as

$$
\begin{aligned}
\Delta_{\epsilon} \int_{\gamma_{1234}} \Phi \varphi= & \int_{\gamma_{1234}} \Phi \varphi_{12} \otimes \int_{\gamma_{125}} \Phi \varphi+\int_{\gamma_{1234}} \Phi \varphi_{23} \otimes \int_{\gamma_{235}} \Phi \varphi \\
& +\int_{\gamma_{1234}} \Phi \varphi_{34} \otimes \int_{\gamma_{345}} \Phi \varphi+\int_{\gamma_{1234}} \Phi \varphi_{14} \otimes \int_{\gamma_{145}} \Phi \varphi .
\end{aligned}
$$


We explicitly computed the 16 entries of the period matrix $P(\vec{\gamma} ; \vec{\varphi} ; \Phi)$ and checked that the coaction

$$
\Delta_{\epsilon} P_{I J}(\vec{\gamma} ; \vec{\varphi} ; \Phi)=\sum_{i} P_{I i}(\vec{\gamma} ; \vec{\varphi} ; \Phi) \otimes P_{i J}(\vec{\gamma} ; \vec{\varphi} ; \Phi)
$$

satisfies the relation in eq. (4.2) order by order in $\epsilon$ through weight 4 .

In all previous examples, we were always able to write all the entries in the coaction in terms of the same class of function. For example, the coaction of Gauss' hypergeometric function ${ }_{2} F_{1}$ only involves ${ }_{2} F_{1}$ functions (and Gamma functions), cf. e.g., eq. (5.14). It is therefore natural to ask if this can still be achieved for the entries in eq. (7.26), i.e., if all integrals that appear in eq. (7.26) can be written in terms of Appell $F_{2}$ functions. This is true, as shown for example in [63]. We now present a brief argument for why this must be the case.

Consider the period matrix for the Appell $F_{2}$ functions. We recall that, in our conventions (see eq. (3.17)), elements in each row have the same integration contour and elements in each column have the same integrand (without loss of generality, we choose the forms in eq. (7.25) as generators of the cohomology group). To simplify the argument we include $\gamma_{1234}$ in our basis of cycles. Then, all elements of the corresponding row are explicitly Appell $F_{2}$ functions. Equivalently, in each column there is at least one entry that is explicitly an $F_{2}$ function. We now recall that Appell $F_{2}$ functions satisfy a given system of secondorder differential equations, see e.g. [61]. For each column (i.e., for each integrand), there is a different set of differential operators that annihilate the corresponding $F_{2}$ function. Since the differential operator is independent of the contour, it in fact annihilates all elements in the column. We conclude that all elements of each column are (linear combinations of) $F_{2}$ functions, since they all satisfy the Appell $F_{2}$ differential equations. We note that the four elements in each column span the space of solutions of the corresponding system of differential equations.

Combined with eq. (7.27), the previous considerations imply that all entries in the coaction can be written in terms of Appell $F_{2}$ functions. It is, however, not obvious how to achieve this in practice, and we were not able to find any change of variables that allows us to express the integrals in eq. (7.26) in terms of $F_{2}$ functions. At this point we recall that the integrals depend on the cycles only through their homology classes, i.e., equivalence classes of cycles that differ by boundaries, which integrate to zero. We are thus free to replace the basis of cycles by any other basis for the homology group without changing the space of integrals that they generate. An alternative basis for the homology group associated to the Appell $F_{2}$ function was constructed in ref. [64]. We denote this basis in the following by $\vec{\Gamma}=\left(\Gamma_{\emptyset}, \Gamma_{1}, \Gamma_{2}, \Gamma_{12}\right)$, where $\Gamma_{\emptyset}=\gamma_{1234}$ as defined in eq. (7.24). ${ }^{9}$ For details of how the cycles are constructed, we refer to section 4 of ref. [64]. Here, we simply quote the result of Theorem 4.4 of ref. [64], which makes it explicit that the integrals on these cycles can be written in terms of $F_{2}$. Let $\omega$ denote the generic integrand

$$
\omega=u^{\beta} v^{\beta^{\prime}}(1-u)^{\gamma-\beta-1}(1-v)^{\gamma^{\prime}-\beta^{\prime}-1}(1-x u-y v)^{-\alpha} d \log u \wedge d \log v .
$$

\footnotetext{
${ }^{9}$ We have adapted the notation in ref. [64] and set $\Gamma_{\emptyset}=\Delta$ and $\Gamma_{I}=\Delta_{I}$.
} 
Then,

$$
\begin{aligned}
\int_{\Gamma_{\emptyset}} \omega= & \frac{\Gamma(\beta) \Gamma(\gamma-\beta) \Gamma\left(\beta^{\prime}\right) \Gamma\left(\gamma^{\prime}-\beta^{\prime}\right)}{\Gamma(\gamma) \Gamma\left(\gamma^{\prime}\right)} F_{2}\left(\alpha ; \beta, \beta^{\prime} ; \gamma, \gamma^{\prime} ; x, y\right) \\
\int_{\Gamma_{1}} \omega= & -e^{i \pi(\beta-\gamma)} x^{1-\gamma} \frac{\Gamma(1-\alpha) \Gamma(\gamma-1) \Gamma\left(\beta^{\prime}\right) \Gamma\left(\gamma^{\prime}-\beta^{\prime}\right)}{\Gamma(\gamma-\alpha) \Gamma\left(\gamma^{\prime}\right)} \\
& \times F_{2}\left(\alpha-\gamma+1 ; \beta-\gamma+1, \beta^{\prime} ; 2-\gamma, \gamma^{\prime} ; x, y\right) \\
\int_{\Gamma_{2}} \omega= & -e^{i \pi\left(\beta^{\prime}-\gamma^{\prime}\right)} y^{1-\gamma^{\prime}} \frac{\Gamma(1-\alpha) \Gamma(\beta) \Gamma(\gamma-\beta) \Gamma\left(\gamma^{\prime}-1\right)}{\Gamma(\gamma) \Gamma\left(\gamma^{\prime}-\alpha\right)} \\
& \times F_{2}\left(\alpha-\gamma^{\prime}+1 ; \beta, \beta^{\prime}-\gamma^{\prime}+1 ; \gamma, 2-\gamma^{\prime} ; x, y\right) \\
\int_{\Gamma_{12}} \omega= & e^{i \pi\left(\beta^{\prime}+\beta-\gamma^{\prime}-\gamma\right)} x^{1-\gamma} y^{1-\gamma^{\prime}} \frac{\Gamma(1-\alpha) \Gamma(\gamma-1) \Gamma\left(\gamma^{\prime}-1\right)}{\Gamma\left(\gamma+\gamma^{\prime}-\alpha-1\right)} \\
& \times F_{2}\left(\alpha-\gamma^{\prime}-\gamma+2 ; \beta-\gamma+1, \beta^{\prime}-\gamma^{\prime}+1 ; 2-\gamma, 2-\gamma^{\prime} ; x, y\right)
\end{aligned}
$$

Since the cycles in $\vec{\Gamma}$ are not constructed as linear combinations of bounded chambers, it is not trivial to relate them to the cycles in $\vec{\gamma}$. However, since they generate the same (twisted) homology group, the period matrices $P(\vec{\gamma} ; \vec{\varphi} ; \Phi)$ and $P(\vec{\Gamma} ; \vec{\varphi} ; \Phi)$ must be related by a linear transformation of the form $P(\vec{\gamma} ; \vec{\varphi} ; \Phi)=K \cdot P(\vec{\Gamma} ; \vec{\varphi} ; \Phi)$. We find that the linear transformation takes the form ${ }^{10}$

$$
K=\left(\begin{array}{cccc}
\frac{c_{3} c_{4}}{\left(c_{1}+c_{3}\right)\left(c_{2}+c_{4}\right)} & \frac{c_{4}}{c_{2}+c_{4}} & \frac{c_{3}}{c_{1}+c_{3}} & 1 \\
-\frac{c_{1} c_{4}}{\left(c_{1}+c_{3}\right)\left(c_{2}+c_{4}\right)} & \frac{c_{4}}{c_{2}+c_{4}} & -\frac{c_{1}}{c_{1}+c_{3}} & 1 \\
\frac{c_{1} c_{2}}{\left(c_{1}+c_{3}\right)\left(c_{2}+c_{4}\right)} & -\frac{c_{2}}{c_{2}+c_{4}} & -\frac{c_{1}}{c_{1}+c_{3}} & 1 \\
-\frac{c_{2} c_{3}}{\left(c_{1}+c_{3}\right)\left(c_{2}+c_{4}\right)} & -\frac{c_{2}}{c_{2}+c_{4}} & \frac{c_{3}}{c_{1}+c_{3}} & 1
\end{array}\right)
$$

Using the change-of-basis matrix $K$, it is straightforward to rewrite the second entry of each tensor of the coaction (7.26) on the $F_{2}$ function in terms of $F_{2}$ functions, as we have done for all other functions discussed above.

The Appell $F_{2}$ function is the special case with $n=2$ of the Lauricella series $F_{A}^{(n)}$, and both versions of the construction of the coaction generalize straightforwardly to all $n$. The twisted cycles of ref. [64] are constructed for arbitrary values of $n$.

\subsection{The Appell $F_{4}$ function}

For completeness, in this section we discuss the Appell $F_{4}$ function. Unlike our previous examples of Appell functions, there is no known integral representation with a sufficiently generic description in terms of hyperplanes. For example, there is an Euler-type represen-

\footnotetext{
${ }^{10} \mathrm{Up}$ to phases, the matrix $K$ is the intersection matrix of the cycles $\vec{\gamma}$ and $\vec{\Gamma}$ [64]. These phases are not relevant for our purpose because the right entry of the coaction on MPLs is defined modulo powers of $\pi$, and thus eq. (4.2) holds irrespectively of these factors.
} 
tation with linear factors, given by

$$
\begin{aligned}
& F_{4}\left(\alpha, \beta, \gamma, \gamma^{\prime} ; x(1-y), y(1-x)\right)=\frac{\Gamma(\gamma) \Gamma\left(\gamma^{\prime}\right)}{\Gamma(\alpha) \Gamma(\beta) \Gamma(\gamma-\alpha) \Gamma\left(\gamma^{\prime}-\beta\right)} \\
& \quad \times \int_{0}^{1} d u \int_{0}^{1} d v u^{\alpha-1} v^{\beta-1}(1-u)^{\gamma-\alpha-1}(1-v)^{\gamma^{\prime}-\beta-1} \\
& \quad \times(1-u x)^{\alpha-\gamma-\gamma^{\prime}+1}(1-v y)^{\beta-\gamma-\gamma^{\prime}+1}(1-u x-v y)^{\gamma+\gamma^{\prime}-\alpha-\beta-1} .
\end{aligned}
$$

However, while the $F_{4}$ function depends on four parameters $\alpha, \beta, \gamma$ and $\gamma^{\prime}$, the integrand has singularities located on 7 hyperplanes, so the exponents of the different factors are not independent. Moreover, although the seven factors appearing in this representation represent hyperplanes, the arrangement is said to be degenerate, because there are points at which three hyperplanes intersect simultaneously (the points $(u, v)=(0,1 / y)$ and $(1 / x, 0)$, also called non-normal crossings).

To discuss this example along the lines of the previous cases, we could consider a larger class of functions where each of the seven factors in the integrand of eq. (7.31) is raised to a different power, and then take the limit corresponding to the $F_{4}$ function. This approach indeed leads to a coaction on the $F_{4}$ functions but, similarly to the case of the $F_{2}$ family, in this representation it is not obvious that all entries of the coaction are $F_{4}$ functions.

Instead, we follow a more direct route and use the basis of cycles and integrands proposed in ref. [65]. The starting point is the Kummer representation

$$
\begin{aligned}
& \int_{\gamma_{1}} t_{1}^{\beta-\gamma} t_{2}^{\beta-\gamma^{\prime}} L\left(t_{1}, t_{2}\right)^{\gamma+\gamma^{\prime}-\alpha-2} Q\left(t_{1}, t_{2}, x, y\right)^{-\beta} d t_{1} d t_{2}= \\
& \quad=\frac{\Gamma(1-\gamma) \Gamma\left(1-\gamma^{\prime}\right) \Gamma\left(\gamma+\gamma^{\prime}-\alpha-1\right)}{\Gamma(1-\alpha)} F_{4}\left(\alpha, \beta, \gamma, \gamma^{\prime} ; x(1-y), y(1-x)\right),
\end{aligned}
$$

where

$$
L\left(t_{1}, t_{2}\right)=1-t_{1}-t_{2}, \quad Q\left(t_{1}, t_{2}, x_{1}, x_{2}\right)=t_{1} t_{2}-x(1-y) t_{2}-y(1-x) t_{1}
$$

and the integration region $\gamma_{1}$ is a twisted version of the region bounded by $L\left(t_{1}, t_{2}\right)$ and $Q\left(t_{1}, t_{2}, x_{1}, x_{2}\right)$. This representation features four hypersurfaces, matching the number of exponents, but the polynomial $Q\left(t_{1}, t_{2}, x_{1}, x_{2}\right)$ is quadratic in the $t_{i}$. For a precise description of $\gamma_{1}$ and the remaining elements of the basis of twisted cycles, $\gamma_{2}, \gamma_{3}$, and $\gamma_{4}$, we refer to ref. [65]. ${ }^{11}$ For a generic integrand

$$
\omega=t_{1}^{\beta-\gamma} t_{2}^{\beta-\gamma^{\prime}} L\left(t_{1}, t_{2}\right)^{\gamma+\gamma^{\prime}-\alpha-2} Q\left(t_{1}, t_{2}, x, y\right)^{-\beta} d t_{1} d t_{2}
$$

\footnotetext{
${ }^{11}$ In that paper, the twisted cycles are denoted by $\Delta_{i}$ rather than $\gamma_{i}$. We have changed the notation in this section to fit with our own conventions.
} 
the integrals over the basis of twisted cycles are then given by [65]

$$
\begin{aligned}
\int_{\gamma_{1}} \omega= & \frac{\Gamma(1-\gamma) \Gamma\left(1-\gamma^{\prime}\right) \Gamma\left(\gamma+\gamma^{\prime}-\alpha-1\right)}{\Gamma(1-\alpha)} F_{4}\left(\alpha, \beta, \gamma, \gamma^{\prime} ; x(1-y), y(1-x)\right), \\
\int_{\gamma_{2}} \omega= & \frac{\Gamma(\alpha+1-\gamma) \Gamma(\beta+1-\gamma) \Gamma(1-\beta) \Gamma\left(\gamma+\gamma^{\prime}-\alpha-1\right)}{\Gamma(2-\gamma) \Gamma\left(\gamma^{\prime}\right)} e^{i \pi\left(\alpha+\beta-\gamma-\gamma^{\prime}\right)}(x(1-y))^{1-\gamma} \\
& F_{4}\left(\alpha-\gamma+1, \beta-\gamma+1,2-\gamma, \gamma^{\prime} ; x(1-y), y(1-x)\right), \\
\int_{\gamma_{3}} \omega= & \frac{\Gamma\left(\alpha+1-\gamma^{\prime}\right) \Gamma\left(\beta+1-\gamma^{\prime}\right) \Gamma(1-\beta) \Gamma\left(\gamma+\gamma^{\prime}-\alpha-1\right)}{\Gamma(\gamma) \Gamma\left(2-\gamma^{\prime}\right)} e^{i \pi\left(\alpha+\beta-\gamma-\gamma^{\prime}\right)}(y(1-x))^{1-\gamma^{\prime}} \\
& F_{4}\left(\alpha-\gamma^{\prime}+1, \beta-\gamma^{\prime}+1, \gamma, 2-\gamma^{\prime} ; x(1-y), y(1-x)\right), \\
\int_{\gamma_{4}} \omega= & \frac{\Gamma(\gamma-1) \Gamma\left(\gamma^{\prime}-1\right) \Gamma(1-\beta)}{\Gamma\left(\gamma+\gamma^{\prime}-\beta-1\right)}(x(1-y))^{1-\gamma}(y(1-x))^{1-\gamma^{\prime}} \\
& F_{4}\left(\alpha-\gamma-\gamma^{\prime}+2, \beta-\gamma-\gamma^{\prime}+2,2-\gamma, 2-\gamma^{\prime} ; x(1-y), y(1-x)\right) .
\end{aligned}
$$

As a basis of integrands, we adapt the basis of ref. [65] so that it is explicitly given by $d$ log-forms:

$$
\begin{aligned}
& \phi_{1}=d \log \frac{t_{1}}{L\left(t_{1}, t_{2}\right)} \wedge d \log \frac{t_{2}}{L\left(t_{1}, t_{2}\right)}=\frac{d t_{1} \wedge d t_{2}}{t_{1} t_{2} L\left(t_{1}, t_{2}\right)}, \\
& \phi_{2}=d \log t_{2} \wedge d \log L\left(t_{1}, t_{2}\right)=\frac{d t_{1} \wedge d t_{2}}{t_{2} L\left(t_{1}, t_{2}\right)}, \\
& \phi_{3}=-d \log t_{1} \wedge d \log L\left(t_{1}, t_{2}\right)=\frac{d t_{1} \wedge d t_{2}}{t_{1} L\left(t_{1}, t_{2}\right)}, \\
& \phi_{4}=d \log \frac{t_{1}-x}{t_{1}-1+y} \wedge d \log \frac{Q\left(t_{1}, t_{2}, x_{1}, x_{2}\right)}{L\left(t_{1}, t_{2}\right)}=(1-x-y) \frac{d t_{1} \wedge d t_{2}}{L\left(t_{1}, t_{2}\right) Q\left(t_{1}, t_{2}, x, y\right)} .
\end{aligned}
$$

Finally, we define the twist

$$
\Phi=t_{1}^{(b-c) \epsilon} t_{2}^{\left(b-c^{\prime}\right) \epsilon} L\left(t_{1}, t_{2}\right)^{\left(c+c^{\prime}-a\right) \epsilon} Q\left(t_{1}, t_{2}, x, y\right)^{-b \epsilon},
$$

and compute the period matrix $P(\vec{\gamma} ; \vec{\phi} ; \Phi)$. Using eq. (3.24) we obtain ${ }^{12}$

$C(\Omega(\vec{\gamma}) ; \vec{\phi} ; \Phi)=\frac{1}{\epsilon^{2}}\left(\begin{array}{cccc}\frac{a}{c c^{\prime}\left(a-c-c^{\prime}\right)} & \frac{1}{c^{\prime}\left(a-c-c^{\prime}\right)} & \frac{1}{c\left(a-c-c^{\prime}\right)} & \frac{a}{c c^{\prime}\left(a-c-c^{\prime}\right)} \\ \frac{1}{(c-b)\left(c+c^{\prime}-a\right)} & 0 & \frac{c^{\prime}}{(c-a)(c-b)\left(a-c-c^{\prime}\right)} & \frac{1}{b\left(a-c-c^{\prime}\right)} \\ \frac{1}{\left(c^{\prime}-b\right)\left(c+c^{\prime}-a\right)} & \frac{c}{\frac{1}{\left(c^{\prime}-a\right)\left(c^{\prime}-b\right)\left(a-c-c^{\prime}\right)}} & 0 & \frac{1}{b\left(a-c-c^{\prime}\right)} \\ \frac{1}{c c^{\prime}} & 0 & 0 & \frac{b-c-c^{\prime}}{b c c^{\prime}}\end{array}\right)$.

We can then construct a new basis of forms $\vec{\varphi}$ from $\vec{\varphi}=\vec{\phi}^{T} \cdot C^{-1}(\Omega(\vec{\gamma}) ; \vec{\phi} ; \Phi)$, such that $P(\vec{\gamma} ; \vec{\varphi} ; \Phi)=\mathbb{1}_{4}+\mathcal{O}(\epsilon)$. We do not write an explicit expression for the new basis of forms $\vec{\varphi}$ as it is lengthy and not illuminating.

\footnotetext{
${ }^{12}$ We note that the cycles in $\vec{\gamma}$ are not obviously positive geometries, and in principle we do not know how to compute the associated canonical forms. Nevertheless, as was done explicitly in the previous section, we can rewrite $\vec{\gamma}$ in terms of positive geometries and then compute $\Omega(\vec{\gamma})$. Here we use a shortcut and directly compute the matrix of intersection numbers from the period matrix, which is possible because we use $d \log$ forms as generators of the cohomology group.
} 
For a generic integrand $\omega=\Phi \varphi$ with $\varphi$ in the cohomology group generated by the forms in $\vec{\varphi}$, we then find that

$$
\Delta_{\epsilon} \int_{\gamma_{1}} \omega=\int_{\gamma_{1}} \Phi \varphi_{1} \otimes \int_{\gamma_{1}} \omega+\int_{\gamma_{1}} \Phi \varphi_{2} \otimes \int_{\gamma_{2}} \omega+\int_{\gamma_{1}} \Phi \varphi_{3} \otimes \int_{\gamma_{3}} \omega+\int_{\gamma_{1}} \Phi \varphi_{4} \otimes \int_{\gamma_{4}} \omega .
$$

We have checked that eq. (4.2) is satisfied for all the entries of the period matrix $P(\vec{\gamma} ; \vec{\varphi} ; \Phi)$ through weight 4 by explicit calculation.

The Appell $F_{4}$ function is the special case with $n=2$ of the Lauricella series $F_{C}^{(n)}$, but since this is a more complicated positive geometry, it is not so clear how to identify bases suitable for generalizing the construction of the coaction to $n>2$. The bases of ref. [65] are given specifically for Appell $F_{4}$.

\section{The generalized hypergeometric function ${ }_{p+1} F_{p}$}

The last example that we will explore is a class of integrals related to the hypergeometric functions ${ }_{p+1} F_{p}$. This function has a recursive Euler-type integral representation,

$$
\begin{aligned}
& { }_{p+1} F_{p}\left(\alpha_{1}, \ldots, \alpha_{p+1} ; \beta_{1}, \ldots, \beta_{p} ; x\right)=\frac{\Gamma\left(\beta_{p}\right)}{\Gamma\left(\alpha_{p+1}\right) \Gamma\left(\beta_{p}-\alpha_{p+1}\right)} \\
& \int_{0}^{1} u^{\alpha_{p+1}-1}(1-u)^{\beta_{p}-\alpha_{p+1}-1}{ }_{p} F_{p-1}\left(\alpha_{1}, \ldots, \alpha_{p} ; \beta_{1}, \ldots, \beta_{p-1} ; x u\right) d u
\end{aligned}
$$

beginning with the trivial case ${ }_{1} F_{0}(\alpha ; x)=(1-x)^{-\alpha}$.

To construct the coaction on the hypergeometric function ${ }_{p+1} F_{p}$ we consider the differential forms

$$
\omega_{p}=d u_{1} \wedge \ldots \wedge d u_{p}\left(1-x \prod_{i=1}^{p} u_{i}\right)^{q+c \epsilon} \prod_{i=1}^{p} u_{i}^{n_{i}+a_{i} \epsilon}\left(1-u_{i}\right)^{m_{i}+b_{i} \epsilon} .
$$

with $n_{i}, m_{i}, q \in \mathbb{R}$ and generic $a_{i}, b_{i}, c \in \mathbb{C}^{*}$. We define the twist $\Phi$ in the usual way,

$$
\Phi=\left(1-x \prod_{i=1}^{p} u_{i}\right)^{c \epsilon} \prod_{i=1}^{p} u_{i}^{a_{i} \epsilon}\left(1-u_{i}\right)^{b_{i} \epsilon}
$$

In the following we assume that the range of all products is from 1 to $p$ and do not write it explicitly. Up to a normalization factor that only involves beta functions, the hypergeometric function ${ }_{p+1} F_{p}$ is given by the integral

$$
f_{p}(x)=\int_{\gamma_{1, \ldots, p}} \omega_{p}
$$

where $\gamma_{1, \ldots, p}=\left\{\left(u_{1}, \ldots, u_{p}\right) \in \mathbb{R}^{p} \mid 0<u_{i}<1\right\}$.

It is easy to determine that the dimensions of the (co)homology groups associated with this class of integrals is $p+1$. In direct analogy to what was done in the case of the ${ }_{2} F_{1}$ 
(see eq. (5.9)), as a basis of the cohomology group we choose the $d \log$-forms

$$
\begin{aligned}
\phi_{1, \ldots, p} & =d u_{1} \wedge \ldots \wedge d u_{p} \prod_{i} \frac{1}{1-u_{i}}, \\
\phi_{1, \ldots, j, \ldots, c} & =d u_{1} \wedge \ldots \wedge d u_{p} \frac{1}{1-x \prod_{i} u_{i}} \prod_{i \neq j} \frac{1}{1-u_{i}} .
\end{aligned}
$$

As a basis of the homology group we choose the cycles

$$
\begin{aligned}
\gamma_{1, \ldots, p} & =\left\{\left(u_{1}, \ldots, u_{p}\right) \in \mathbb{R}^{p} \mid 0<u_{i}<1\right\}, \\
\gamma_{1, \ldots, \hat{j}, \ldots, p, c} & =\left\{\left(u_{1}, \ldots, u_{p}\right) \in \mathbb{R}^{p} \mid 0<u_{i}<1, i \neq j ; 0<u_{j}<x^{-1} \prod_{i \neq j} u_{i}^{-1}\right\} .
\end{aligned}
$$

We can then compute the period matrix $P(\vec{\gamma} ; \vec{\phi} ; \Phi)$ to find that

$$
P(\vec{\gamma} ; \vec{\phi} ; \Phi)=\frac{1}{\epsilon^{p}} \mathcal{M}+\mathcal{O}\left(\epsilon^{p-1}\right),
$$

where $\mathcal{M}=\operatorname{diag}\left(d_{0}^{p}, \ldots, d_{p}^{p}\right)$ with

$$
d_{0}^{p}=\prod_{i} b_{i}^{-1}, \quad d_{j}^{p}=\frac{1}{x c} \prod_{i \neq j} \frac{a_{j}+b_{j}-a_{i}-b_{i}}{b_{i}\left(a_{j}+b_{j}-a_{i}\right)} \quad \text { for } j \geq 1 .
$$

Relying on the relation given in eq. (3.24), this determines the matrix of intersection numbers $C(\Omega(\vec{\gamma}) ; \vec{\phi} ; \Phi)$. It is then trivial to define a new basis $\vec{\varphi}$ of forms such that $C(\Omega(\vec{\gamma}) ; \vec{\varphi} ; \Phi)=\mathbb{1}_{p+1}$ :

$$
\varphi_{1, \ldots, p}=\frac{\epsilon^{p}}{d_{0}^{p}} \phi_{1, \ldots, p}, \quad \varphi_{1, \ldots, \hat{j}, \ldots, p, c}=\frac{\epsilon^{p}}{d_{j}^{p}} \phi_{1, \ldots, \hat{j}, \ldots, p, c} .
$$

As a result, the coaction can be cast in the form

$$
\Delta_{\epsilon} \int_{\gamma_{1, \ldots, p}} \omega_{p}=\int_{\gamma_{1, \ldots, p}} \Phi \varphi_{1, \ldots, p} \otimes \int_{\gamma_{1, \ldots, p}} \omega_{p}+\sum_{j=1}^{p} \int_{\gamma_{1, \ldots, p}} \varphi_{1, \ldots, \hat{j}, \ldots, p, c} \otimes \int_{\gamma_{1, \ldots, \hat{j}, \ldots, p, c}} \omega_{p} .
$$

To obtain the coaction on a ${ }_{p+1} F_{p}$ function we simply need to normalize the above expression by a product of beta functions. One can easily check that this expression reduces to the coaction on the ${ }_{2} F_{1}$ we constructed in section 5 for $p=1$. We have also checked explicitly the case $p=2$, corresponding to the ${ }_{3} F_{2}$ hypergeometric function.

We finish by noting that all integrals in the period matrix $P(\vec{\gamma} ; \vec{\varphi} ; \Phi)$, and thus in the coaction eq. (8.10), can be written in terms of ${ }_{p+1} F_{p}$ functions [63]. For instance, for $p=2$ this can be done with the relation

$$
\begin{array}{rl}
\int_{0}^{1} d u \int_{0}^{1 / x u} & d v u^{\alpha_{3}-1}(1-u)^{\beta_{2}-\alpha_{3}-1} v^{\alpha_{2}-1}(1-v)^{\beta_{1}-\alpha_{2}-1}(1-x u v)^{-\alpha_{1}}= \\
& \frac{\Gamma\left(\alpha_{2}\right) \Gamma\left(1-\beta_{1}\right) \Gamma\left(\alpha_{3}\right) \Gamma\left(\beta_{2}-\alpha_{3}\right)}{\Gamma\left(\alpha_{2}-\beta_{1}+1\right) \Gamma\left(\beta_{2}\right)}(-1)^{\alpha_{2}}{ }_{3} F_{2}\left(\alpha_{1}, \alpha_{2}, \alpha_{3} ; \beta_{1}, \beta_{2} ; x\right) \\
& +\frac{\Gamma\left(1-\alpha_{1}\right) \Gamma\left(\beta_{1}-1\right) \Gamma\left(\alpha_{3}-\beta_{1}+1\right) \Gamma\left(\beta_{2}-\alpha_{3}\right)}{\Gamma\left(\beta_{1}-\alpha_{1}\right) \Gamma\left(\beta_{2}-\beta_{1}+1\right)}(-1)^{\alpha_{2}-\beta_{1}+1} x^{1-\beta_{1}} \\
& \times{ }_{3} F_{2}\left(\alpha_{1}-\beta_{1}+1, \alpha_{2}-\beta_{1}+1, \alpha_{3}-\beta_{1}+1 ; 2-\beta_{1}, \beta_{2}-\beta_{1}+1 ; x\right),
\end{array}
$$

which can be generalized to an arbitrary ${ }_{p+1} F_{p}$. 


\section{Summary and discussion}

In this paper we have introduced a coaction $\Delta_{\epsilon}$ on large classes of hypergeometric functions, for which the coefficients of the Laurent expansion in $\epsilon$ involve only polylogarithmic functions. In particular, we restrict ourselves to cases where we can find a basis of the homology group associated to the integral such that for each homology generator there is a unique $d \log$-form with singularities on its boundaries. A convenient setting to realize this condition is to consider positive geometries, and all the examples considered in this paper fall into this class. Once an appropriate basis of the (co)homology groups has been identified, we can easily write down the coaction by computing the entries of the period matrix and the matrix of intersection numbers. The main property of our coaction is that it is consistent with expanding the functions in $\epsilon$, i.e., acting with $\Delta_{\epsilon}$ and then expanding each factor in $\epsilon$ is equivalent to first expanding in $\epsilon$ and then computing the coaction of the MPLs in the Laurent coefficients.

We have illustrated our coaction on various hypergeometric functions, in particular on ${ }_{p+1} F_{p}$ and Appell functions, with generalizations to the Lauricella series $F_{A}, F_{B}$, and $F_{D}$. Application to other hypergeometric functions whose integral representations consist of a product of linear factors raised to generic exponents expanded around integers (hyperplane arrangements) is completely straightforward. This class includes many of the LauricellaSaran functions [14] such as $F_{N}$ and $F_{S}$.

Since many Feynman integrals, at one-loop and beyond, can be expressed in terms of these functions, it will be interesting to connect our results to the recently proposed coaction on Feynman integrals [5, 9, 10]. The coaction on one-loop integrals is by now understood, but the generalization of the results of ref. [10] beyond one loop is still an open problem. The results of this paper can be used to explore the extension of the coaction to two-loop Feynman integrals and beyond, as seen for example in ref. [34].

It is important to note that the hypergeometric functions appearing in known Feynman integrals actually violate a key assumption in the results related to twisted (co)homology and intersection numbers, namely that the exponents in the integral representations (the $a_{I}$ of (3.2)) are nonzero and independent. For this reason, we have emphasized that we are able to derive valid coaction formulas in degenerate limits of the ${ }_{2} F_{1}$, and we have argued that a detailed analysis of twisted cycles allows such limits to be taken in general. Although it was not discussed in this paper, we have checked that we obtain consistent coactions for degenerations of more complicated hypergeometric functions that appear in one-loop integrals. These were all found to be in agreement with the diagrammatic coaction of ref. [10]. We remark that a similar degenerate limit was taken in ref. [67] in the context of computing intersection numbers in period integrals associated to K3 surfaces.

Our main formula for the coaction is a conjecture, and it would be interesting to prove it rigorously. First steps in this direction have recently been taken in ref. [36], albeit in the restricted case of one-dimensional integrals. Inspired by our preliminary results (see, e.g., conference talks [33-35]), the authors of ref. [36] have studied in detail the family of Lauricella functions $F_{D}^{(n)}$ considered in section 6. In particular, they have defined a motivic version of this family of Lauricella functions, and they were able to show that it is 
possible to define a coaction on the Lauricella function $F_{D}^{(n)}$ which is consistent with the (motivic) coaction on MPLs after expansion in $\epsilon$. The coaction obtained from the motivic setup, however, is not directly comparable to the coaction defined here, as we now explain. We have already argued that the second factor in the coaction should not change under analytic continuation, and we therefore only consider MPLs modulo their discontinuities in the second entry, i.e., only MPLs modulo $2 \pi i$. Alternatively, one can interpret the objects in the second entry as single-valued versions of hypergeometric functions and MPLs [4]. This is the approach taken in ref. [36], where all Lauricella functions and MPLs in the second entry are the single-valued versions of these functions. It would be interesting to see how this alternative choice of representing the second entries in the coaction compares to our formula, and in particular what is the role played by the matrix of intersection numbers. Here we only mention that the matrix of intersection numbers is closely connected to the computation of single-valued functions, as was for example pointed out in the context of string amplitudes $[4,29,52,57,68]$. It would be fascinating to explore this connection further, and put our conjectured coaction on a rigorous mathematical ground, at least for the case of the Lauricella functions considered in ref. [36].

Our coaction construction is quite general, but we have considered it explicitly on certain named hypergeometric functions with well-known integral representations. Because the second entries of our coaction are constructed by integrating over each of the basis elements of twisted homology, these integrals are not necessarily easy to recognize as belonging to the same class of function. We have argued that they must satisfy the same differential equation, and the methods of refs. [64, 65, 69] may lead to expressions in which this property is manifest, when desired. However, we do not know whether the twisted cycles constructed in these methods have canonical forms of their own. We have discussed this issue in section 7.3 for the Appell $F_{2}$ function, where we were able to deduce a relation between the twisted cycles of ref. [64] and the homology classes constructed from bounded chambers. It may also be desirable to consider when it is possible to select bases with sparse matrices of intersection numbers, in order to minimize the number of terms in the coaction formula, as we have done for example for ${ }_{2} F_{1}$. Diagonal matrices of intersection numbers have been constructed for example in ref. [70].

Other interesting avenues for future research would be to see if the coaction defined here can be extended to more general classes of hypergeometric integrals. In particular, here we have restricted ourselves to the study of hypergeometric functions whose expansion in $\epsilon$ only involves MPLs. Since, in the motivic setting, the coaction on MPLs is a special case of the coaction on motivic periods (see, e.g., ref. [4]), it would be interesting to understand if it is likewise possible to extend our coaction to hypergeometric functions that involve more general periods than MPLs as Laurent coefficients.

\section{Acknowledgments}

The authors are grateful to Nima Arkani-Hamed, Francis Brown, Clement Dupont, Benjamin Enriquez, Javier Fresan, Riccardo Gonzo, Yoshiako Goto, Martijn Hidding, SaieiJaeyong Matsubara-Heo, Sebastian Mizera, Erik Panzer, and Johann Usovitsch for dis- 
cussions. The authors acknowledge the hospitality of the Galileo Galilei Institute (GGI), Florence, and of the Institute for Theoretical Studies (ITS) of the ETH Zurich during the programmes "Amplitudes in the LHC era" and "Modular Forms, Periods and Scattering Amplitudes." SA, CD, EG and JM wish to thank Trinity College Dublin and its Hamilton Mathematics Institute for hospitality, and SA, RB and CD acknowledge the hospitality of the Higgs Center for Theoretical Physics of the University of Edinburgh, at various stages of this work. SA wishes to thank the Centre for High Energy Physics at McGill University, Montréal, for hospitality. RB also wishes to thank the Institute for Advanced Study, Princeton, for hospitality. EG wishes to thank the CERN theory department for hospitality as a Scientific Associate. This work is supported by the "Fonds National de la Recherche Scientifique" (FNRS), Belgium (SA), by the ERC Consolidator Grant 647356 "CutLoops" (RB), the ERC Starting Grant 637019 "MathAm" (CD), and the STFC Consolidated Grant "Particle Physics at the Higgs Centre" (EG, JM).

Open Access. This article is distributed under the terms of the Creative Commons Attribution License (CC-BY 4.0), which permits any use, distribution and reproduction in any medium, provided the original author(s) and source are credited.

\section{References}

[1] P. Belkale and P. Brosnan, Periods and Igusa local zeta functions, Int. Math. Res. Not. 2003 (2003) 2655 [math/0302090].

[2] C. Bogner and S. Weinzierl, Periods and Feynman integrals, J. Math. Phys. 50 (2009) 042302 [arXiv: 0711.4863] [INSPIRE].

[3] M. Kontsevich and D. Zagier, Periods, in Mathematics unlimited - 2001 and beyond, B. Engquist and W. Schmid eds., Springer, Heidelberg Germany (2001), pg. 771.

[4] F. Brown, Notes on Motivic Periods, Commun. Num. Theor. Phys. 11 (2017) 557 [arXiv: 1512.06410].

[5] F. Brown, Feynman amplitudes, coaction principle and cosmic Galois group, Commun. Num. Theor. Phys. 11 (2017) 453 [arXiv:1512.06409] [INSPIRE].

[6] E. Panzer and O. Schnetz, The Galois coaction on $\phi^{4}$ periods, Commun. Num. Theor. Phys. 11 (2017) 657 [arXiv: 1603.04289] [INSPIRE].

[7] O. Schnetz, The Galois coaction on the electron anomalous magnetic moment, Commun. Num. Theor. Phys. 12 (2018) 335 [arXiv:1711.05118] [INSPIRE].

[8] S. Caron-Huot, L.J. Dixon, F. Dulat, M. Von Hippel, A.J. McLeod and G. Papathanasiou, The Cosmic Galois Group and Extended Steinmann Relations for Planar $\mathcal{N}=4 S Y M$ Amplitudes, JHEP 09 (2019) 061 [arXiv:1906.07116] [INSPIRE].

[9] S. Abreu, R. Britto, C. Duhr and E. Gardi, Algebraic Structure of Cut Feynman Integrals and the Diagrammatic Coaction, Phys. Rev. Lett. 119 (2017) 051601 [arXiv:1703.05064] [INSPIRE].

[10] S. Abreu, R. Britto, C. Duhr and E. Gardi, Diagrammatic Hopf algebra of cut Feynman integrals: the one-loop case, JHEP 12 (2017) 090 [arXiv:1704.07931] [INSPIRE]. 
[11] D. Fotiadi and F. Pham, Analytic Properties of Some Integrals over Complex Manifolds, in Homology and Feynman integrals, R.C. Hwa and V.L. Teplitz eds., W.A. Benjamin Inc., New York U.S.A. (1966).

[12] S. Abreu, R. Britto, C. Duhr and E. Gardi, Cuts from residues: the one-loop case, JHEP 06 (2017) 114 [arXiv: 1702.03163] [INSPIRE].

[13] A. Erdelyi, Higher Transcendental Functions. Vol. 1, McGraw-Hill, New York U.S.A. (1953).

[14] S. Saran, Transformations of certain hypergeometric functions of three variables, Acta Math. 93 (1955) 293.

[15] J. Fleischer, F. Jegerlehner and O.V. Tarasov, A New hypergeometric representation of one loop scalar integrals in d dimensions, Nucl. Phys. B 672 (2003) 303 [hep-ph/0307113] [INSPIRE].

[16] A.I. Davydychev, Four-point function in general kinematics through geometrical splitting and reduction, J. Phys. Conf. Ser. 1085 (2018) 052016 [arXiv:1711.07351] [inSPIRE].

[17] B.A. Kniehl and O.V. Tarasov, Analytic result for the one-loop scalar pentagon integral with massless propagators, Nucl. Phys. B 833 (2010) 298 [arXiv:1001.3848] [INSPIRE].

[18] O.V. Tarasov, Hypergeometric representation of the two-loop equal mass sunrise diagram, Phys. Lett. B 638 (2006) 195 [hep-ph/0603227] [INSPIRE].

[19] V.V. Bytev, M. Yu. Kalmykov and B.A. Kniehl, Differential reduction of generalized hypergeometric functions from Feynman diagrams: One-variable case, Nucl. Phys. B 836 (2010) 129 [arXiv:0904.0214] [INSPIRE].

[20] B.A. Kniehl and O.V. Tarasov, Finding new relationships between hypergeometric functions by evaluating Feynman integrals, Nucl. Phys. B 854 (2012) 841 [arXiv:1108.6019] [INSPIRE].

[21] J. Blümlein, K.H. Phan and T. Riemann, Scalar one-loop vertex integrals as meromorphic functions of space-time dimension d, Acta Phys. Polon. B 48 (2017) 2313 [arXiv: 1711.05510] [INSPIRE].

[22] K.H. Phan and T. Riemann, Scalar 1-loop Feynman integrals as meromorphic functions in space-time dimension d, Phys. Lett. B 791 (2019) 257 [arXiv:1812.10975] [InSPIRE].

[23] K.H. Phan and D.T. Tran, One-loop three-point Feynman integrals with Appell $F_{1}$ hypergeometric functions, PTEP 2019 (2019) 063B01 [arXiv: 1904.07430] [INSPIRE].

[24] L. de la Cruz, Feynman integrals as A-hypergeometric functions, JHEP 12 (2019) 123 [arXiv: 1907.00507] [INSPIRE].

[25] R.P. Klausen, Hypergeometric Series Representations of Feynman Integrals by GKZ Hypergeometric Systems, arXiv:1910.08651 [INSPIRE].

[26] N. Arkani-Hamed, Y. Bai and T. Lam, Positive Geometries and Canonical Forms, JHEP 11 (2017) 039 [arXiv: 1703.04541] [INSPIRE].

[27] K. Aomoto and M. Kita, Theory of Hypergeometric Functions, Springer Monographs in Mathematics, Springer Japan, Tokyo Japan (2011).

[28] S. Mizera, Scattering Amplitudes from Intersection Theory, Phys. Rev. Lett. 120 (2018) 141602 [arXiv: 1711.00469] [INSPIRE].

[29] S. Mizera, Aspects of Scattering Amplitudes and Moduli Space Localization, Ph.D. thesis, Perimeter Inst. Theor. Phys., 2019. arXiv: 1906.02099 [INSPIRE]. 
[30] P. Mastrolia and S. Mizera, Feynman Integrals and Intersection Theory, JHEP 02 (2019) 139 [arXiv: 1810.03818] [INSPIRE].

[31] H. Frellesvig et al., Decomposition of Feynman Integrals on the Maximal Cut by Intersection Numbers, JHEP 05 (2019) 153 [arXiv:1901.11510] [INSPIRE].

[32] H. Frellesvig, F. Gasparotto, M.K. Mandal, P. Mastrolia, L. Mattiazzi and S. Mizera, Vector Space of Feynman Integrals and Multivariate Intersection Numbers, Phys. Rev. Lett. 123 (2019) 201602 [arXiv: 1907.02000] [INSPIRE].

[33] S. Abreu, R. Britto, C. Duhr, E. Gardi and J. Matthew, Coaction for Feynman integrals and diagrams, PoS (LL2018) 047 (2018) [arXiv:1808.00069] [INSPIRE].

[34] S. Abreu, R. Britto, C. Duhr and E. Gardi, The diagrammatic coaction and the algebraic structure of cut Feynman integrals, PoS (RADCOR2017) 002 (2018) [arXiv:1803.05894] [INSPIRE].

[35] S. Abreu, R. Britto, C. Duhr, E. Gardi and J. Matthew, A coaction on generalised hypergeometric functions, talk at Modular Forms, Periods and Scattering Amplitudes, ETH Zurich, Zurich Switzerland (2019).

[36] F. Brown and C. Dupont, Lauricella hypergeometric functions, unipotent fundamental groups of the punctured Riemann sphere and their motivic coactions, arXiv:1907.06603 [INSPIRE].

[37] A.B. Goncharov, Multiple polylogarithms and mixed Tate motives, math/0103059.

[38] C. Duhr, Mathematical aspects of scattering amplitudes, in Proceedings of Theoretical Advanced Study Institute in Elementary Particle Physics: Journeys Through the Precision Frontier: Amplitudes for Colliders (TASI 2014), Boulder U.S.A. (2014), pg. 419 [arXiv: 1411.7538] [INSPIRE].

[39] A.B. Goncharov, Galois symmetries of fundamental groupoids and noncommutative geometry, math/0208144.

[40] F.C.S. Brown, Mixed Tate Motives over $\mathbb{Z}$, Ann. Math. 175 (2012) 949.

[41] F. Brown, On the decomposition of motivic multiple zeta values, in Advanced Studies in Pure Mathematics. Vol. 68: Galois-Teichmüller theory and arithmetic geometry, Math. Soc. Japan, Tokyo Japan (2012), pg. 31 [arXiv:1102.1310] [INSPIRE].

[42] F. Brown, private communication (2019).

[43] S. Moch, P. Uwer and S. Weinzierl, Nested sums, expansion of transcendental functions and multiscale multiloop integrals, J. Math. Phys. 43 (2002) 3363 [hep-ph/0110083] [INSPIRE].

[44] S. Moch and P. Uwer, XSummer: Transcendental functions and symbolic summation in form, Comput. Phys. Commun. 174 (2006) 759 [math-ph/0508008] [INSPIRE].

[45] S. Weinzierl, Symbolic expansion of transcendental functions, Comput. Phys. Commun. 145 (2002) 357 [math-ph/0201011] [INSPIRE].

[46] T. Huber and D. Maître, HypExp: A Mathematica package for expanding hypergeometric functions around integer-valued parameters, Comput. Phys. Commun. 175 (2006) 122 [hep-ph/0507094] [INSPIRE].

[47] M. Yu. Kalmykov, Gauss hypergeometric function: Reduction, $\epsilon$-expansion for integer/half-integer parameters and Feynman diagrams, JHEP 04 (2006) 056 [hep-th/0602028] [INSPIRE]. 
[48] J. Ablinger, J. Blümlein and C. Schneider, Analytic and Algorithmic Aspects of Generalized Harmonic Sums and Polylogarithms, J. Math. Phys. 54 (2013) 082301 [arXiv:1302.0378] [INSPIRE].

[49] Z. Bern, V. Del Duca, W.B. Kilgore and C.R. Schmidt, The infrared behavior of one loop QCD amplitudes at next-to-next-to leading order, Phys. Rev. D 60 (1999) 116001 [hep-ph/9903516] [INSPIRE].

[50] R.N. Lee and A.A. Pomeransky, Critical points and number of master integrals, JHEP 11 (2013) 165 [arXiv:1308.6676] [INSPIRE].

[51] T. Bitoun, C. Bogner, R.P. Klausen and E. Panzer, The number of master integrals as Euler characteristic, PoS (LL2018) 065 (2018) [arXiv: 1809.03399] [INSPIRE].

[52] F. Brown and C. Dupont, Single-valued integration and double copy, arXiv:1810.07682 [INSPIRE].

[53] K. Matsumoto, Intersection numbers for logarithmic k-forms, Osaka J. Math. 35 (1998) 873.

[54] J.M. Drummond and É. Ragoucy, Superstring amplitudes and the associator, JHEP 08 (2013) 135 [arXiv: 1301.0794] [INSPIRE].

[55] O. Schlotterer and S. Stieberger, Motivic Multiple Zeta Values and Superstring Amplitudes, J. Phys. A 46 (2013) 475401 [arXiv:1205.1516] [InSPIRE].

[56] C.R. Mafra and O. Schlotterer, One-loop open-string integrals from differential equations: all-order $\alpha^{\prime}$-expansions at $n$ points, arXiv:1908.10830 [INSPIRE].

[57] S. Mizera, Combinatorics and Topology of Kawai-Lewellen-Tye Relations, JHEP 08 (2017) 097 [arXiv: 1706 . 08527] [INSPIRE].

[58] M. Abramowitz and A.S. Irene, Handbook of Mathematical Functions, Dover Publications, Mineola U.S.A. (1972).

[59] C. Duhr and F. Dulat, PolyLogTools — polylogs for the masses, JHEP 08 (2019) 135 [arXiv: 1904.07279] [INSPIRE].

[60] K. Matsumoto, Relative twisted homology and cohomology groups associated with Lauricella's $F_{D}$, arXiv: 1804.00366

[61] Gradshteyn and Ryzhik, Table of Integrals, Series and Products, Academic Press, New York U.S.A. (1943).

[62] G. Lauricella, Sulle funzioni ipergeometriche a piu variabili, Rend. Circ. Mat. Palermo 7 (1893) 111.

[63] K. Mimachi and M. Noumi, Solutions in terms of integrals of multivalued functions for the classical hypergeometric equations and the hypergeometric system on the configuration space, Kyushu J. Math. 70 (2016) 315.

[64] Y. Goto, Twisted period relations for Lauricella's hypergeometric functions $F_{A}$, Osaka J. Math. 07861 [arXiv:1310.6088].

[65] Y. Goto and K. Matsumoto, The monodromy representation and twisted period relations for Appell's hypergeometric function $F_{4}$, Nagoya Math. J. 217 (2015) 61 [arXiv:1310.4243].

[66] V.V. Bytev, M. Yu. Kalmykov and B.A. Kniehl, HYPERDIRE, HYPERgeometric functions DIfferential REduction: MATHEMATICA-based packages for differential reduction of generalized hypergeometric functions ${ }_{p} F_{p-1}, F_{1}, F_{2}, F_{3}, F_{4}$, Comput. Phys. Commun. 184 (2013) 2332 [arXiv: 1105.3565] [inSPIRE]. 
[67] S.-J. Matsubara-Heo and N. Takayama, An algorithm of computing cohomology intersection number of hypergeometric integrals, arXiv:1904.01253.

[68] F. Brown and C. Dupont, Single-valued integration and superstring amplitudes in genus zero, arXiv: 1910.01107 [INSPIRE].

[69] Y. Goto, Twisted cycles and twisted period relations for Lauricella's hypergeometric function $F_{c}$, Int. J. Math. 24 (2013) [arXiv: 1308.5535].

[70] K. Matsumoto, Pfaffian of Lauricella's hypergeometric system $F_{A}$, arXiv:1502.00334. 